\title{
Is it impossible to acquire absolute pitch in adulthood?
}

\author{
Yetta Kwailing Wong ${ }^{1}$ - Kelvin F. H. Lui ${ }^{2}$ Ken H. M. Yip ${ }^{2}$ - Alan C.-N. Wong ${ }^{2}$ (D)
}

Published online: 4 November 2019

(C) The Psychonomic Society, Inc. 2019

\begin{abstract}
Absolute pitch (AP) refers to the rare ability to name the pitch of a tone without external reference. It is widely believed to be only for the selected few with rare genetic makeup and early musical training during the critical period, and therefore acquiring AP in adulthood is impossible. Previous studies have not offered a strong test of the effect of training because of issues like small sample size and insufficient training. In three experiments, adults learned to name pitches in a computerized, gamified and personalized training protocol for 12 to 40 hours, with the number of pitches gradually increased from three to twelve. Across the three experiments, the training covered different octaves, timbre, and training environment (inside or outside laboratory). AP learning showed classic characteristics of perceptual learning, including generalization of learning dependent on the training stimuli, and sustained improvement for at least one to three months. $14 \%$ of the participants ( 6 out of 43 ) were able to name twelve pitches at $90 \%$ or above accuracy, comparable to that of 'AP possessors' as defined in the literature. Overall, AP continues to be learnable in adulthood, which challenges the view that AP development requires both rare genetic predisposition and learning within the critical period. The finding calls for reconsideration of the role of learning in the occurrence of AP, and pushes the field to pinpoint and explain the differences, if any, between the aspects of AP more trainable in adulthood and the aspects of AP that are potentially exclusive for the few exceptional AP possessors observed in the real world.
\end{abstract}

Keywords Absolute pitch $\cdot$ Pitch perception $\cdot$ Musical training $\cdot$ Perceptual learning $\cdot$ Perceptual expertise $\cdot$ Critical period

Absolute pitch (AP) refers to the ability to name the pitch of a tone (e.g., naming a tone as "C") or to produce it without external reference tones (Takeuchi \& Hulse, 1993; W. D. Ward, 1999). While the majority of us can effortlessly identify a countless number of faces, objects, and visual and auditory words, most people find it very difficult to name the twelve pitches, and professional musicians are no exception (Athos et al., 2007; Levitin \& Rogers, 2005; Zatorre, 2003). The most extreme estimate states that, in every 10,000 people, there is one 'AP possessor' who can perform AP judgment accurately

Electronic supplementary material The online version of this article (https://doi.org/10.3758/s13414-019-01869-3) contains supplementary material, which is available to authorized users.

Yetta Kwailing Wong

yetta.wong@gmail.com

$\bowtie \quad$ Alan C.-N. Wong

alanwong@cuhk.edu.hk

1 Department of Educational Psychology, Faculty of Education, The Chinese University of Hong Kong, Rm 308, Ho Tim Building Shatin N.T. Hong Kong

2 Department of Psychology, The Chinese University of Hong Kong, 344 Sino Building Shatin N.T. Hong Kong and effortlessly (Takeuchi \& Hulse, 1993). This rare ability is considered a special talent and endowment for gifted musicians (Deutsch, 2002; Takeuchi \& Hulse, 1993; W. D. Ward, 1999; but see Levitin \& Rogers, 2005). The genesis of AP has therefore been a perplexing research topic among musicians, psychologists and neuroscientists for more than a century (Deutsch, 2002; Levitin \& Rogers, 2005; Takeuchi \& Hulse, 1993; W. D. Ward, 1999).

At the cognitive level, a widely accepted hypothesis suggests that AP involves two stages of processing (Levitin, 1994; Levitin \& Rogers, 2005). The first stage is AP memory, which general listeners can also establish through exposure to tones, songs and music excerpts (Halpern, 1989; Levitin, 1994; Schellenberg \& Trehub, 2003). This pitch memory is typically regarded as implicit, i.e., participants cannot verbally describe what has been remembered, and as absolute because participants can discriminate between original musical excerpts and the excerpts that were shifted in pitch by one semitone without the assistance of external pitch references (Schellenberg \& Trehub, 2003). The second stage is the ability to associate the represented AP memory with verbal labels, which is somehow mastered by the 'AP possessors' only (Brancucci, Dipinto, Mosesso, \& Tommasi, 2009; Deutsch, 2002; Levitin \& Rogers, 2005; Schellenberg \& Trehub, 
2003; Vanzella \& Schellenberg, 2010). In other words, the bottleneck of AP performance is in assigning verbal labels to the tones rather than establishing AP memory representation per se.

At the neural level, AP has been associated with distinct neural markers. In functional neural imaging, 'AP possessors' have shown an increased activity in the superior temporal gyrus (Ohnishi et al., 2001; Schulze, Gaab, \& Schlaug, 2009; Wengenroth et al., 2013; S. J. Wilson, Lusher, Wan, Dudgeon, \& Reutens, 2009) and the left dorsal lateral prefrontal cortex (Bermudez \& Zatorre, 2005; Zatorre, Perry, Beckett, Westbury, \& Evans, 1998). Individuals with and without AP have also been shown to differ in their brain activations reflected in various event-related potentials (ERPs), including the N1 (Itoh, Suwazono, Arao, Miyazaki, \& Nakada, 2005; Pantev et al., 1998; Wu, Kirk, Hamm, \& Lim, 2008), P2a (Wengenroth et al., 2013), and P3 (Hantz, Crummer, Wayman, Walton, \& Frisina, 1992; Hirose et al., 2002; Klein, Coles, \& Donchin, 1984; Rogenmoser, Elmer, \& Jäncke, 2015). Other structural and functional connectivity differences have also been identified, including the size asymmetry of the planum temporale (Keenan, Thangaraj, Halpern, \& Schlaug, 2001; Schlaug, Jancke, Huang, \& Steinmetz, 1995), the architecture of the superior longitudinal fasciculus (Oechslin, Imfeld, Loenneker, Meyer, \& Jäncke, 2009), and functional connectivity of different brain regions (Jäncke, Langer, \& Hänggi, 2012; Loui, Li, Hohmann, \& Schlaug, 2011; Loui, Zamm, \& Schlaug, 2012). These suggest that AP may be supported by both functional and structural brain differences in 'AP possessors', as compared with other listeners.

\section{The role of genes and the critical period}

How can we explain the development of AP in 'AP possessors'? One influential theory suggests that AP develops if two prerequisites are fulfilled (Bachem, 1940; Baharloo, Johnston, Service, Gitschier, \& Freimer, 1998; Chin, 2003; Drayna, 2007; Levitin \& Rogers, 2005; Ross, Olson, Marks, \& Gore, 2004; Takeuchi \& Hulse, 1993; Trainor, 2005; Zatorre, 2003). The first prerequisite is the rare genetic disposition to AP. It is reported that AP runs in families, as siblings of 'AP possessors' are more likely to be 'AP possessors' (about 14 - 48\% of the 'AP possessors' reported to have a sibling or first-degree relatives that was also an 'AP possessor'; Baharloo et al., 1998; Baharloo, Service, Risch, Gitschier, \& Freimer, 2000; Gregersen, Kowalsky, Kohn, \& Marvin, 1999, 2001). AP is often considered a relatively 'clean' cognitive phenotype (Baharloo et al., 2000; Gregersen et al., 2001), and some proposed that AP may be subserved by a single gene (Drayna,
2007; but see Theusch, Basu, \& Gitschier, 2009 which suggests that AP is genetically heterogeneous).

The second prerequisite is an early onset of musical training that is within the critical period in childhood. A strong definition of critical period refers to the early period of life during which experience is essential for normal development and leads to permanent changes in brain functions and behavior (Knudsen, 2004) ${ }^{1}$, and we based the current discussion on this definition because this description fits well with the widespread belief of AP (Bachem, 1940; Baharloo et al., 1998; Chin, 2003; Drayna, 2007; Levitin \& Rogers, 2005; Ross et al., 2004; Takeuchi \& Hulse, 1993; Trainor, 2005; Zatorre, 2003). The critical period of AP is thought to be similar to that of language development (Chin, 2003; Deutsch, Dooley, Henthorn, \& Head, 2009). Supporting evidence comes from large-scale survey studies in which the average onset of musical training of 'AP possessors' was 5.4 years, about 2.5 years earlier than that of 'non-AP possessors' (Gregersen et al., 1999; see also Baharloo et al., 1998; Gregersen, Kowalsky, Kohn, \& Marvin, 2001).

Importantly, AP training was considered relatively successful in young children (Crozier, 1997; Miyazaki \& Ogawa, 2006; Sakakibara, 2014). In contrast, while AP can improve to some extent in adulthood with deliberate practice (Brady, 1970; Cuddy, 1968, 1970; Hartman, 1954; Meyer, 1899; Mull, 1925; Russo, Windell, \& Cuddy, 2003; Van Hedger, Heald, Koch, \& Nusbaum, 2015; Wedell, 1934), there is no convincing evidence that adults can attain a performance level comparable to 'AP possessors' through training (Bachem, 1940; Levitin \& Rogers, 2005; W. D. Ward, 1999). According to this influential theory, most professional musicians fail to acquire AP because they fail to start their music training within the critical period and/or because they do not carry the specific genes. Training AP in adulthood, when the critical period of acquiring AP has long passed, should be practically impossible (Bachem, 1940; Crozier, 1997; Trainor, 2005; but see Gervain et al., 2013 for the possibility of reopening the critical period by taking a drug).

Interestingly, the concept of critical period well explains the development of basic functions and structure of different sensory systems but not for high-level cognitive abilities. In vision, depriving the visual input into one eye led to reduced ocular dominance in the visual cortex of young cats but not in deprived adult cats (Hubel \& Wiesel, 1970; Wiesel \& Hubel, 1963). In hearing, disrupting sound input led to abnormal development of the auditory cortex in the early life of rats but not in older rats (Zhang, Bao, \& Merzenich, 2002). In touch, depriving tactile inputs after birth led to abnormal

\footnotetext{
${ }^{1}$ A weaker definition of critical period refers to the early period of life during which experience has a particularly strong effect on development than the same experience at other times, while plasticity may still be observable and extend to adulthood (e.g., Hooks \& Chen, 2007; Sengpiel, 2007). This definition makes the concept undifferentiable from 'sensitive period'.
} 
functions of the somatosensory cortex (Simons \& Land, 1987). Overall, normal development of the sensory cortices is determined by the environmental inputs during the early period of life (but see Hooks \& Chen, 2007 for recent challenges of this view).

However, evidence is less as clear for high-level cognitive abilities. In vision, higher-level visual abilities, such as acuity, stereopsis and crowding, continue to develop in adulthood (Daw, 1998). In musical development, various degrees of plasticity in adulthood have been observed during the acquisition of musical pitch structure (e.g., consonance and dissonance, scale structure and harmonics) and the functional specialization of the auditory and motor cortices (see review in Trainor, 2005). In language acquisition, while it has been argued that there is a critical period for acquiring a native-like accent (Patkowski, 1990; Scovel, 1988), subsequent research demonstrated that it is possible for later learners, who started to acquire a second language after twelve years old, to attain native accents in various languages, including English, French and Dutch (Flege, Munro, \& MacKay, 1995; see review in Singleton, 2001). In synesthesia, in which the perception of a stimulus consistently evokes experience that is not physically present (e.g., seeing the color 'red' on a black letter; J. Ward, 2013), the proposed cause is reduced pruning during early childhood within the critical period (Maurer \& Mondloch, 2006). While synesthesia has a close genetic link with AP (Gregersen et al., 2013), synesthetic experiences can also be learned in adulthood (Bor, Rothen, Schwartzman, Clayton, \& Seth, 2015). In sum, different complex human behaviors, previously thought to be constrained by the critical period, are now shown to be learnable in adulthood. AP seems to stand as an interesting exception that its development is suggested to be constrained by the critical period (Zeanah, Gunnar, McCall, Kreppner, \& Fox, 2011).

A closer look into the literature of AP indicates that direct evidence for a critical period constraining AP development is weak. Similar to accent acquisition, some cases of AP were identified with later onset of musical training in the normal population (about 3-4\% in the age groups of 9-12 and beyond 12; Baharloo et al., 1998) and in individuals with the Williams Syndrome (3 out of 5 'AP possessors' with the Williams Syndrome started musical training at 8 years old or later; Lenhoff, Perales, \& Hickok, 2001). These suggest that early onset of musical training during the critical period is not essential for developing AP (Gregersen et al., 2001). If the critical period is not essential and the genetic contribution of AP is only moderate, these two factors are unlikely to be the whole picture that describes AP development (Baharloo et al., 1998, 2000; Gregersen et al., 1999, 2001). What other factors could explain the genesis of AP?

\section{The role of experience}

A possible alternative is that AP is developed through perceptual learning. Perceptual learning refers to the long-term improvement on a perceptual task as a result of perceptual experience (Fahle \& Poggio, 2002; Goldstone, 1998; Sasaki, Nanez, \& Watanabe, 2010). It has been repeatedly demonstrated that humans extract information from environmental inputs and fine-tune their perceptual representations accordingly in different sensory modalities, including the visual (Fiorentini \& Berardi, 1980; Karni \& Sagi, 1993), auditory (Fujioka, Ross, Kakigi, Pantev, \& Trainor, 2006; Kraus \& Banai, 2007), somatosensory (Sathian \& Zangaladze, 1997) and olfactory domains (D. A. Wilson \& Stevenson, 2003). Perceptual learning occurs even when the stimuli are irrelevant to the task at hand (Seitz \& Watanabe, 2009), for the nondiagnostic and task-irrelevant information of the trained stimuli (Y. K. Wong, Folstein, \& Gauthier, 2011), and when participants are unaware of the information carried by the stimuli (Tsushima, Sasaki, \& Watanabe, 2006; Watanabe, Nanez, \& Sasaki, 2001). These demonstrate how the perceptual system is constantly tuned to environmental inputs.

Consistent with the perceptual learning hypothesis of AP, it is well-established that AP is shaped by experience (Takeuchi \& Hulse, 1993; Y. K. Wong \& Wong, 2014). For example, better pitch naming is often observed in musicians when the testing conditions better match their prior experience, such as using tones in the timbre of one's own instrument (see review in Takeuchi \& Hulse, 1993), tones in a highly used pitch like 'A4', which is the standard tuning pitch in orchestras (Levitin \& Rogers, 2005; Takeuchi \& Hulse, 1993), tones presented in a multisensory testing context similar to one's musical training (Y. K. Wong \& Wong, 2014), and tones associated with the more frequently used white keys than black keys (Athos et al., 2007; Miyazaki, 1989, 1990; Takeuchi \& Hulse, 1993). Even performance in 'AP possessors' can be disrupted by recent listening experience with detuned music (Hedger, Heald, \& Nusbaum, 2013). Experience also explains why nonmusicians can recognize the starting tones of songs or melodies that are highly familiar in an absolute manner, which indicates that non-musicians form absolute memory of tunes based on repeated exposure (Levitin, 1994; Schellenberg \& Trehub, 2003).

Previous training studies might have failed to convince researchers that AP is trainable in adulthood because of various issues. For example, training duration was very short in some studies (roughly 1-4 hours; Cuddy, 1970; Mull, 1925; Van Hedger et al., 2015), which might have limited the potential of observing larger effects in AP learning. Some training studies used a very small sample size ( 3 or less participants per condition; Brady, 1970; Cuddy, 1968; Hartman, 1954; Meyer, 1899; Mull, 1925; Wedell, 1934) and involved self-training of the authors of the manuscripts (Brady, 1970; Meyer, 1899). 
Other training studies were difficult to interpret. For example, with only a binary judgment on a single tone learned (e.g. " $\mathrm{C}$ " or not "C"; Mull, 1925; Russo et al., 2003), it is difficult to interpret whether the learned ability is comparable to 'AP' or not. Another study did not provide sufficient information (e.g., details of the testing task after training, accuracy, etc.) for interpreting the performance of the participants (Lundin, 1963). Therefore, despite the apparently substantial AP improvement attained in some of these studies (e.g., Brady, 1970; Lundin, 1963), it remains unclear whether AP can be acquired in adulthood. Furthermore, previous training studies might have lacked key components of effective training regimes, such as computerized stimulus presentation and feedback protocols that enable indiviudalized training progress, incentive for sustaining motivation of the training, etc, which might have collectively limited the potential AP improvement during training.

In this study, we tested whether AP acquisition in adulthood, i.e., attaining a performance level comparable to that of real-world 'AP possessors', is possible through perceptual training. This directly tested whether a critical period of development of AP exists, as stated in the genes and critical period theory of AP. We also aimed to examine whether the training-induced improvements are consistent with that of perceptual learning. This tested whether the perceptual learning theory of AP development can account for the behavioral changes (if any). We defined AP as the ability to name pitches accurately without external references, which is one of the most common definitions of AP in the literature (Levitin \& Rogers, 2005; Takeuchi \& Hulse, 1993; W. D. Ward, 1999). To test whether AP can be acquired, we first needed to define the performance level of real-world 'AP possessors'. However, our survey of the literature revealed that the definition of 'AP possessors' was variable and arbitrary in previous work (e.g., using self-report or different performance-based measurements, with different scoring methods and cut-off points; see Methods). In this study, we arbitrarily adopted a relatively stringent training criterion of AP - being able to name, without any reference, all of the twelve pitches that constitute an octave at $90 \%$ accuracy, with semitone errors considered incorrect. Our survey of the literature indicates that participants who have passed our training would also be considered 'AP possessors' in most of the published AP studies that adopted performance-based definition of 'AP possessors' (see Methods of Experiment 1). This indicates that individuals having passed our training have acquired AP performance comparable to that of 'AP possessors' as defined in the literature.

To address our question, we designed different training regimes of AP by optimizing various factors, including the use of extended training duration, larger sample sizes, modern computerized protocols and measures to provide individualized training and to sustain motivation, e.g., by gamifying the training and including monetary reward for the training progress. In three experiments, we trained 43 adults to name the pitch of tones with different combinations of timbres and octaves for 12-40 hours in laboratory and mobile online settings. If specific genetic disposition and an early onset of music training are essential for AP acquisition, AP training in adulthood should be largely in vain and result in very limited improvement in all participants. Alternatively, if AP can be trained in adulthood as a type of perceptual learning, it should be possible, at least for some individuals, to attain a performance level similar to that of real-world 'AP possessors'.

In addition, if AP is developed through perceptual learning, then AP learning should demonstrate several characteristics that match well with that of perceptual learning studies. First, perceptual learning typically leads to performance enhancement after training (Fahle \& Poggio, 2002; Goldstone, 1998). In this study, we examined whether AP performance improved by measuring the number of pitches that participants learned to name accurately without feedback through training (see Methods).

Second, we examined whether the degree of generalization of AP learning changed as a function of the octaves and timbres involved in training. Although perceptual learning is often regarded as highly specific to the training stimuli (Fahle \& Poggio, 2002; Watanabe \& Sasaki, 2015), especially when the training is difficult and supported by more specific inputs (Ahissar \& Hochstein, 2004), generalization of learning is often present in various untrained conditions to different degrees (Banai \& Lavner, 2014; Fahle \& Poggio, 2002; Watanabe \& Sasaki, 2015; Wright, Buonomano, Mahncke, \& Merzenich, 1997). Instead of simply regarding perceptual learning as 'specific', the degree of learning specificity and generalization during perceptual training is best conceptualized by understanding the psychological space involved in training and testing - whether the testing space overlaps with the training space would affect the extent of generalization (Y. K. Wong et al., 2011; see also Nosofsky, 1986, 1987; Palmeri \& Gauthier, 2004). Hence, we predicted that including training tones in more octaves and timbres (as in Experiment 1 and 3 ) should cover larger regions of the psychological space, and thus lead to better generalization to untrained octaves and timbres. Including training tones in a single octave and timbre only should cover a smaller part of the psychological space (as in Experiment 2) and therefore should result in more specific AP learning.

Third, perceptual enhancement in perceptual learning is relatively long-lasting, often in the time scale of months or even years (Fahle \& Poggio, 2002; Goldstone, 1998; Karni \& Sagi, 1993; Watanabe \& Sasaki, 2015). We tested whether AP improvement sustained for one to three months after the end of training.

Finally, if AP is developed through perceptual experience, it is possible that musicians may develop AP more easily with 
the long-term musical training than non-musicians. Learning AP is about establishing the association between the tones and the pitch names. The long-term exposure to musical tones of musicians have fine-tuned the perceptual representations of tones in the auditory system (Pantev et al., 1998), which may in turn facilitate their development of the mapping between the tones and the pitch names. In Experiment 2, musicians had at least six years of formal music training, while non-musicians had no formal music training or had brief training for less than a year. We explored whether musicians would learn AP more efficiently than non-musicians.

\section{Experiment 1}

In Experiment 1, we tested whether learning AP in adulthood is possible and examined the pattern of AP learning. Since how much training contributes to a sufficiently rigorous training to enable AP acquisition in adulthood is an untested empirical question, we decided to conduct a 12-hour training in this experiment, which was comparable to some previous perceptual learning studies in our laboratory (A. C.-N. Wong, Palmeri, \& Gauthier, 2009; Y. K. Wong et al., 2011).

\section{Methods}

\section{Participants}

Ten adults who were native Cantonese speakers were recruited at City University of Hong Kong and completed the training. They included 2 males and 8 females, who were 23.1 years old on average $(S D=4.50)$. Seven of them were regarded as musicians, as they were formally trained in music for 2-10 years, with the major instrument being piano $(N=5)$, violin $(N=1)$ and flute $(N=1)$. Three were regarded as non-musicians as they were not formally trained with music before. One additional participant dropped out in the middle of the training and was excluded from all analyses. All participants filled out a questionnaire about their music training background, including the musical instruments and the highest ABRSM exam passed, and reported if they regarded themselves as 'AP possessors'. As expected, none considered themselves 'AP possessors' before training (otherwise joining the lengthy training would be pointless). In the test for generalization before training (see below), all participants produced an average pitch-naming error larger than 1 semitone with the training tones. A within-1-semitone error was one of the commonly used definitions for 'AP possessors' in the literature (e.g., Bermudez \& Zatorre, 2009; Crozier, 1997; Loui et al., 2012), indicating that their pitch naming ability was not comparable with that of 'AP possessors' before training. They received monetary compensation for the training and testing. Table 1 shows the number of participants and the design for all experiments.

The sample size was estimated based on a recent AP training study (Van Hedger et al., 2015) using GPower 3.1.9.2. In this study, a large effect size was observed for the training improvement in adults (pretest vs. posttest; $f=1.34$ ). Using the same $f$, the sample size required to detect any training effect at $p=.05$ with a power of 0.95 was 5 participants. To be more conservative, we recruited 10 participants. This sample size was also consistent with that used in previous perceptual training studies (Chung \& Truong, 2013; Y. K. Wong et al., 2011).

\section{Materials \& Stimuli}

The experiment was conducted on personal computers using Matlab (Natick, MA) with the PsychToolbox extension (Brainard, 1997; Pelli, 1997) at the Cognition and Neuroscience Laboratory at City University of Hong Kong. Participants were requested to bring their own earphone to the training and testing. They adjusted the volume to a comfortable level before the training or testing started.

In Experiment 1, 120 tones were used. They were synthetic tones and piano tones in octaves three to six, and violin tones in octaves four to five (see Supplementary Figure 1 for their spectral envelop). The synthetic tones were identical to those in prior AP tests, and were generated by summing a series of sinusoidal waveforms including the fundamental frequency and harmonics (Bermudez, Lerch, Evans, \& Zatorre, 2009). The piano tones were recorded with an electric keyboard (Yamaha S31). The violin tones were recorded by a volunteer violinist in a soundproof room. The precision of the tones was checked during recording by a tuner. The deviation between the actual frequency of the recorded tones and the expected frequency for the equal-tempered scale (with $\mathrm{A} 4=440 \mathrm{~Hz}$ ) ranged from $.012 \%$ to $.765 \%$, with a mean deviation of $.231 \%, S D=.193$.

The sound clips were 32-bit with a sampling rate of $44100 \mathrm{~Hz}$. They were edited in Audacity such that they lasted for 1 second with a 0.1 -second linear onset and 0.1 -second linear offset and were matched with similar perceptual magnitude by ear ${ }^{2}$.

\footnotetext{
2 The average intensity values of the tones were $68.3,81.3$ and $65.1 \mathrm{~dB}$ for the piano, synthetic and violin timbres respectively. The peak intensity values of the tones were $73.1,90.3$ and $77.3 \mathrm{~dB}$ for the three timbres respectively. The mean of the root-mean-squared (RMS) values of the tones were $.055, .258$, and .032 respectively. The intensity and amplitude differences reflected the perceptual differences across timbres, since the synthetic tones were perceived to be softer, and the piano and violin tones louder in general.
} 
Table 1 Details of the training protocols in the three experiments

\begin{tabular}{|c|c|c|c|c|c|c|c|c|}
\hline \multirow[t]{2}{*}{ Experiment } & \multicolumn{2}{|c|}{ Participants } & \multicolumn{4}{|c|}{ Training } & \multicolumn{2}{|c|}{ Test for Generalization } \\
\hline & $\mathrm{N}$ & Mus/Non-Mus & Mode & Duration (Hr) & Octave & Timbre & Octave & Timbre \\
\hline 1 & 10 & $7 / 3$ & $\mathrm{Lab}$ & 12 & 4,5 & synthetic, piano & 3,6 & violin \\
\hline 2 & 22 & $10 / 12$ & Lab & 15 & 4 & synthetic & 5 & piano, violin \\
\hline 3 & 11 & $6 / 5$ & Online & 40 & 4,5 & piano, violin, clarinet & 6 & synthetic \\
\hline
\end{tabular}

$\mathrm{N}$ indicates the number of participants in each experiment. 'Mus/Non-Mus' indicates the number of musicians and non-musicians in each experiment respectively

\section{Absolute pitch training}

The training was gamified and structured into 80 levels, which was organized into ten 8-level parts with an increasing number of pitches (from 3 pitches in the first 8 levels to 12 pitches in the last 8 levels; Fig. 1A). Each eight-level part consisted of four types of levels, which included tones that were progressively richer in timbres (synthetic, or synthetic \& piano) and octaves $(4$, or $4 \&$ $5)$. Tones in different octaves and timbres were introduced gradually into the training so as to break down the learning steps into smaller ones and make the learning more achievable. The order of adding new tones, i.e., tones in new octaves were introduced before new timbres, was arbitrary.

Each of the four types of levels was repeated twice, once with trial-by-trial feedback provided, and then once without feedback (Fig. 1A). If participants achieved $90 \%$ accuracy for a certain level, they would proceed to the next level; otherwise they would stay at the same level (Fig. 2A). The training was completed by finishing 12 hours of training or by passing all 80 levels with $90 \%$ accuracy. Participants finished one hour of training per day. They were trained on at least four days per week and finished the training in three weeks.

For example, participants began the training with three pitches $(\mathrm{E}, \mathrm{F}$ and $\mathrm{F} \#)$. At levels $1-2$, synthetic tones in these three pitches in octave 4 were included, with feedback provided at level 1 and then without feedback at level 2. At levels 3-4, synthetic tones in both octaves 4 and 5 were included with feedback and then without feedback. At levels 5-6, synthetic tones and piano tones in octave 4 were included with feedback and then without feedback. At levels 7-8, synthetic tones and piano tones in octaves 4 and 5 were included with feedback and then without feedback. At the no-feedback levels, participants were not provided with any feedback on the correctness of the tones. These no-feedback levels served as mini milestones for participants' AP performance at $90 \%$ accuracy. If they achieved $90 \%$ accuracy at the $8^{\text {th }}$ level, a new pitch was added into the training set, with which they went through the same 8-level part again. Each level included 20 trials, with tones distributed as evenly as possible among the training pitches, octaves and timbres.

A pitch-naming task was used (Fig. 2A). During each trial, an isolated tone was presented for $1 \mathrm{~s}$. Then, an image showing the pitch-to-key mapping was presented. Participants were required to name the pitch of the presented tone by key press within $5 \mathrm{~s}$. Semitone errors were considered errors in the training. Before each level, participants were allowed to freely listen to sample tones of the training pitches as many times as they preferred before proceeding to the training (Figure 2A). In each one-hour training session, participants might have finished different numbers of training trials depending on their pace of learning (e.g., the amount of time spent on training trials or on sample tone listening).

Normally participants earned one point for a correct answer in each trial. To motivate participants, a special trial that was worth three points randomly appeared with a chance of $1 / 80$. Also, participants were given 1, 2 and 3 tokens if they achieved $60 \%, 75 \%$ and $90 \%$ accuracy at a training level respectively. With ten tokens, participants would obtain a chance to initiate a three-point special trial when preferred. Three chances of initiating these special trials at one level were allowed at most. The special trials did not appear and could not be initiated during no-feedback levels. This ensured that participants performed the no-feedback levels without any scoring assistance.

\section{Definition of AP}

In order to compare the performance of trained AP with that of real-world 'AP possessors', we surveyed the literature of AP on Web of Science on $19^{\text {th }}$ April, 2017 with the term 'absolute pitch' in the topic and identified 110 empirical papers. Unfortunately, there was not a single objective definition of the performance level of 'AP possessors'. Instead, these papers used highly varied definitions of 'AP possessors', including self-report, AP performance measurements, or relative performance on AP tasks between participants (such as 3 
Fig. 1 The organization of the training levels in the three experiments in terms of the task, presence of trial-by-trial feedback, and the octave and timbre of the training tones

\section{(a) Experiment 1}

\begin{tabular}{|c|c|c|c|c|c|c|c|c|c|}
\hline \multirow{2}{*}{\multicolumn{2}{|c|}{\begin{tabular}{l|} 
Test for \\
Generalization
\end{tabular}}} & \multirow{2}{*}{\begin{tabular}{|l|}
12 hours \\
Training \\
\end{tabular}} & \multirow{2}{*}{\multicolumn{2}{|c|}{$\begin{array}{l}\text { Test for } \\
\text { Generalization }\end{array}$}} & \multicolumn{3}{|c|}{ After 1 month } & & \\
\hline & & & & & \multicolumn{3}{|c|}{$\begin{array}{l}\text { Test for } \\
\text { Generalization }\end{array}$} & & \\
\hline \multicolumn{2}{|c|}{$\begin{array}{c}\text { Levels } 1-8 \\
\text { Pitches Trained }=3 \\
(E, F, F \#)\end{array}$} & \multicolumn{2}{|c|}{$\begin{array}{l}\frac{\text { Levels } 9-16}{\text { Pitches Trained }=4} \\
\text { (D\#, E, F, F\#) }\end{array}$} & \multicolumn{2}{|c|}{$\begin{array}{l}\text { Levels } 17-24 \\
\text { Pitches Trained }=5 \\
(D \#, E, F, F \#, G)\end{array}$} & $\cdots \cdots$ & \multicolumn{3}{|c|}{$\begin{array}{c}\frac{\text { Levels } 73-80}{\text { Pitches Trained }=12} \\
\text { (C, C\#, D, D\#, E, F, F\#, G, G\#, } \\
\text { A, A\#, B) }\end{array}$} \\
\hline & Level 1 & Level 2 & Level 3 & Level 4 & Leve & & Level 6 & Level 7 & Level 8 \\
\hline Task & Naming & Naming & Naming & Naming & Nam & & Naming & Naming & Naming \\
\hline Feedback & Yes & No & Yes & No & Ye & & No & Yes & No \\
\hline Octave & 4 & 4 & 4,5 & 4,5 & 4 & & 4 & 4,5 & 4,5 \\
\hline Timbre & Synthetic & Synthetic & Synthetic & Synthetic & $\begin{array}{r}\text { Synth } \\
\text { Piar }\end{array}$ & & $\begin{array}{l}\text { Synthetic; } \\
\text { Piano }\end{array}$ & $\begin{array}{c}\text { Synthetic; } \\
\text { Piano }\end{array}$ & $\begin{array}{c}\text { Synthetic; } \\
\text { Piano }\end{array}$ \\
\hline
\end{tabular}

\section{(b) Experiment 2}

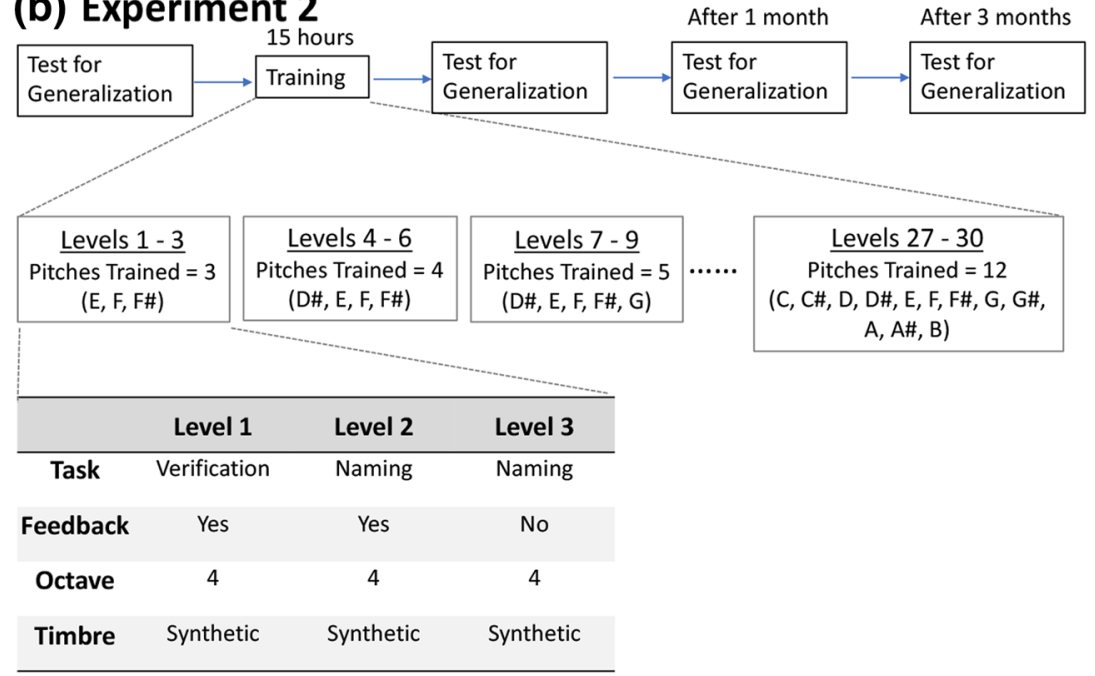

\section{(c) Experiment 3}

\begin{tabular}{|c|c|c|c|c|c|c|c|c|c|}
\hline \multirow{2}{*}{\multicolumn{2}{|c|}{$\begin{array}{l}\text { C) ExperIm } \\
\text { Test for } \\
\text { Generalization } \\
\end{array}$}} & 115 & & & \multicolumn{3}{|c|}{ After 1 month } & \multicolumn{2}{|c|}{ After 3 months } \\
\hline & & Training & \multicolumn{2}{|c|}{$\begin{array}{l}\text { Test for } \\
\text { Generalization }\end{array}$} & & \multicolumn{2}{|c|}{$\begin{array}{l}\text { Test for } \\
\text { Generalization }\end{array}$} & \multicolumn{2}{|c|}{$\begin{array}{l}\text { Test for } \\
\text { Generalization }\end{array}$} \\
\hline \multicolumn{2}{|c|}{$\begin{array}{l}\frac{\text { Levels } 1-8}{\text { Pitches Trained }}=3 \\
(E, F, F \#)\end{array}$} & \multicolumn{2}{|c|}{$\begin{array}{l}\frac{\text { Levels } 9-16}{\text { Pitches Trained }=4} \\
(D \#, E, F, F \#)\end{array}$} & \multicolumn{2}{|c|}{$\begin{array}{c}\frac{\text { Levels } 17-24}{\text { Pitches Trained = }} \\
(D \#, E, F, F \#, G)\end{array}$} & $\cdots \cdots$ & \multicolumn{3}{|c|}{$\begin{array}{c}\text { Levels } 73-80 \\
\text { Pitches Trained = } 12 \\
\text { (C, C\#, D, D\#, E, F, F\#, G, G\#, } \\
\text { A, A\#, B) }\end{array}$} \\
\hline & Level 1 & Level 2 & Level & Level 4 & Lev & el 5 & Level 6 & Level 7 & Level 8 \\
\hline Task & Naming & Naming & Namir & Naming & Nar & ning & Naming & Naming & Naming \\
\hline Feedback & Yes & No & Yes & No & & es & No & Yes & No \\
\hline Octave & 4 & 4 & 4,5 & 4,5 & & 4 & 4 & 4,5 & 4,5 \\
\hline Timbre & Piano & Piano & Pian & Piano & $\begin{array}{l}\text { Pia } \\
\text { Vic } \\
\text { Clar }\end{array}$ & $\begin{array}{l}\text { ano; } \\
\text { olin; } \\
\text { rinet }\end{array}$ & $\begin{array}{l}\text { Piano; } \\
\text { Violin; } \\
\text { Clarinet }\end{array}$ & $\begin{array}{l}\text { Piano; } \\
\text { Violin; } \\
\text { Clarinet }\end{array}$ & $\begin{array}{l}\text { Piano; } \\
\text { Violin; } \\
\text { Clarinet }\end{array}$ \\
\hline
\end{tabular}


Fig. 2 The design of the training levels, training and testing tasks in the three experiments. (A) shows the content of each training level, and the two training tasks. The naming task was used in all experiments. ' $*$ ' indicates that the glissando was adopted in Experiment 2 and 3 only. '\#' indicates that the verification task was adopted in Experiment 2 only. (B) shows the testing task in the test for generalization

\section{(a) AP Training}
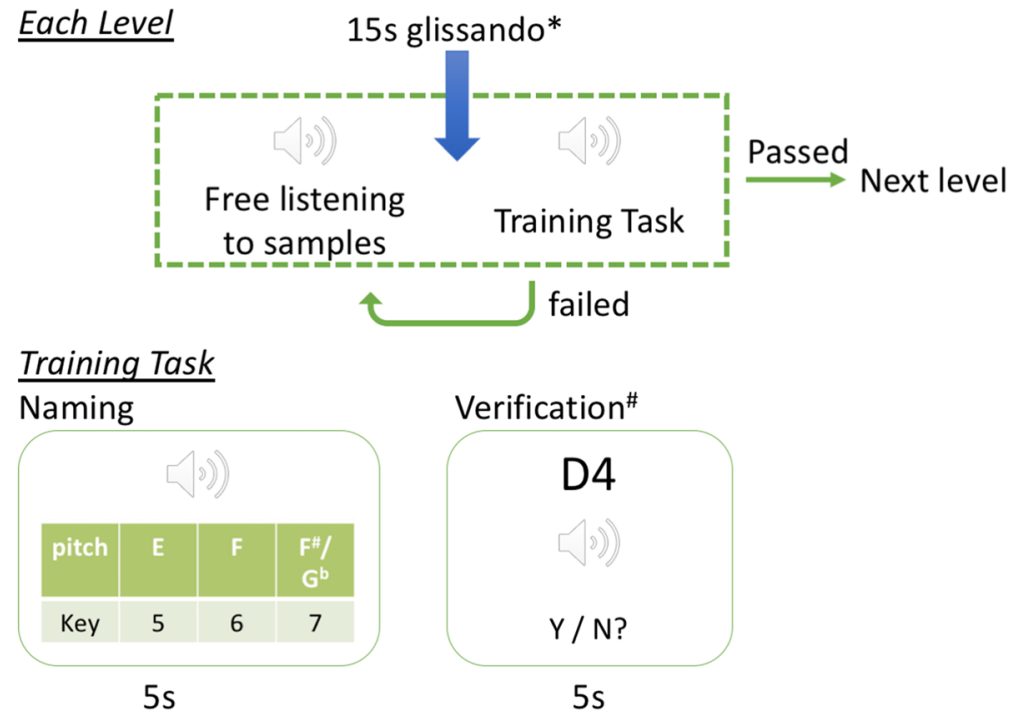

(b) Test for Generalization

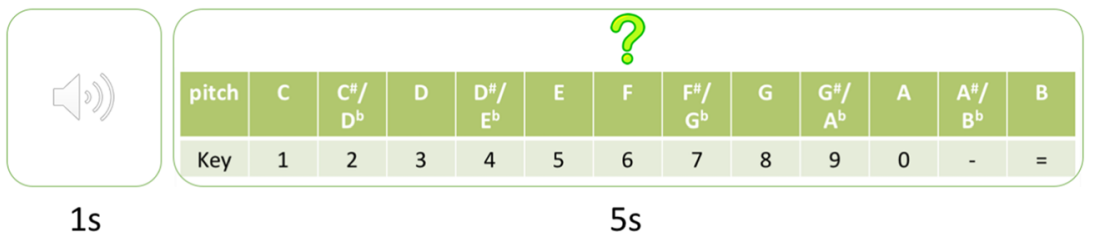

SDs higher in AP accuracy than 'non-AP possessors'). We focused on the 66 publications that defined AP objectively based on AP performance instead of self-report, and found that these papers adopted highly varied performance measures to define 'AP possessors' with different cut-off points and scoring methods (e.g., taking semitone errors as correct, partially correct or incorrect; using accuracy or the average size of errors, etc.).

Given this variability in definition, we did not see any strong reasons to adopt any single definition of 'AP possessors' based on some particular publications. In this study, we arbitrarily adopted a relatively stringent training criterion of AP, i.e., having achieved $90 \%$ pitch naming accuracy with all of the 12 pitches and with semitone errors considered incorrect. We asked whether participants who have passed our training would be considered 'AP possessors' according to the definition specified in each of the 66 papers, and recalculated the participants' performance if needed. Results showed that our successfully trained participants would be considered 'AP possessors' in $83.3 \%$ (55 out of 66) of those papers using objective definitions, meaning that their AP performance was representative of and comparable to that of 'AP possessors' as defined in the literature.

\section{Test for generalization}

The test for generalization was performed before and within three days after training to examine how well the pitchnaming abilities generalized to untrained octaves and timbres (Table 1). An identical test was performed a month later to examine whether the AP learning sustained for at least a month. One hundred and twenty tones in octaves 3 to 6 were used, in which octaves 4 and 5 were trained, and 3 and 6 untrained. Three timbres were included, with synthetic and piano tones as trained timbres, and violin tones as an untrained timbre. The tones were presented in three conditions, either with trained octave and timbre, trained octave and untrained timbre, or untrained octave and trained timbre.

A pitch naming task was used (Fig. 2B). During each trial, a tone was presented for $1 \mathrm{~s}$. Then an image that mapped the 12 pitch names to 12 keys of the keyboard, which was the same as those used in the training, was presented. Participants were required to name the pitch of the presented tone by key press within $5 \mathrm{~s}$. Each tone was presented twice, leading to 240 trials in total. The trials were presented in randomized order. Ten practice trials were provided before testing. The dependent measure was the precision of pitch naming, i.e., the average error in semitone of participants' responses relative to 
the correct responses. A trial without any response will be assigned an error of 3 semitones (the expected error one would have by complete guessing ${ }^{3}$ ). The average percentage of no-response trials in the pretest, posttest and testing after one month was $.066 \%, .027 \%$ and $.27 \%$ respectively ${ }^{4}$. We used the average error instead of the general naming accuracy because measuring the size of judgment errors additionally informs the precision of pitch naming performance of the individuals, which is more informative than the binary correctness of the responses as measured by general naming accuracy.

\section{Results and Discussion}

\section{Learning of AP}

In general, participants made substantial progress in learning to name pitches (Fig. 3A; see individual progress of learning in Supplementary Fig. 2). At the end of training, they were able to name on average 8.1 pitches (out of 12 ; range $=5$ to 12 ; $S D=2.28)$ at $90 \%$ accuracy without any externally provided reference tones under the stringent scoring criterion of taking semitone errors as incorrect.

Importantly, one of the ten participants passed all levels of training, meaning that he was able to name all of the twelve pitches at $90 \%$ accuracy without any externally provided reference tones. With this level of AP performance, he would be recognized as an 'AP possessor' in most of the empirical papers that adopted an objective performance-based definition of AP (see Methods). These suggest that he has acquired AP through perceptual learning in adulthood.

\section{Generalization \& sustainability of AP learning}

The pitch-naming performance improved and was similar between trained and untrained tones (Figure 4A). A 2 x 3 ANOVA with Prepost (pretest / posttest) and Stimulus Type (octave \& timbre trained / octave trained $\&$ timbre untrained / octave untrained \& timbre trained) as factors on pitch naming error revealed a significant main effect of Prepost, $F(1,9)=30.3, p<.001, \eta_{\mathrm{p}}{ }^{2}=$

\footnotetext{
${ }^{3}$ Since the 12 pitches in the Western music system are arranged along a circle, the maximum pitch error is 6 semitones and the minimum error is 0 semitone. Therefore complete guessing (e.g., blind guessing with ' $\mathrm{C}$ ' to be the answer of all trials) would lead to a $(0+1+2+3+4+5+6+5+4+3+2+1) / 12=3$-semitone error on average.

${ }^{4}$ Before training, two participants reported to have absolutely zero knowledge about the name of any tone, and clearly stated that they could only blind guess in the test. They were allowed to skip the test and assigned a chance-level performance, i.e., 3-semitone error for all conditions, and therefore they were excluded from this analysis during pretest.
}

(a) Experiment 1

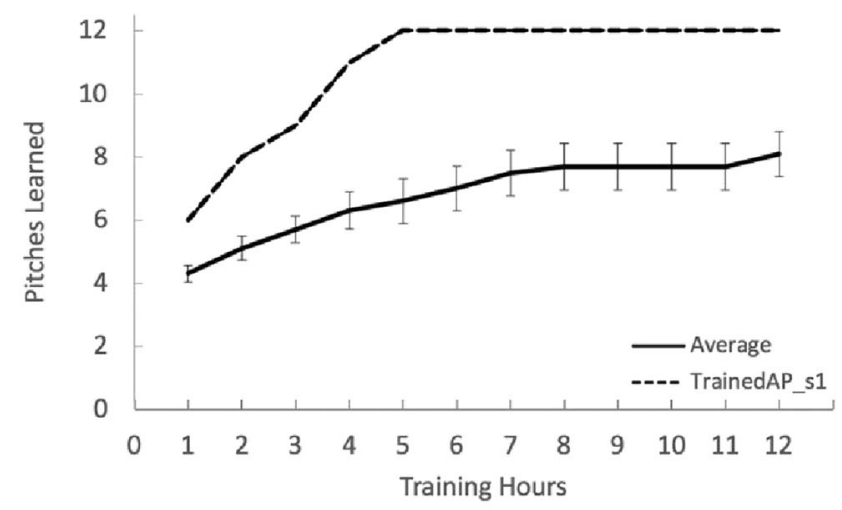

(b) Experiment 2

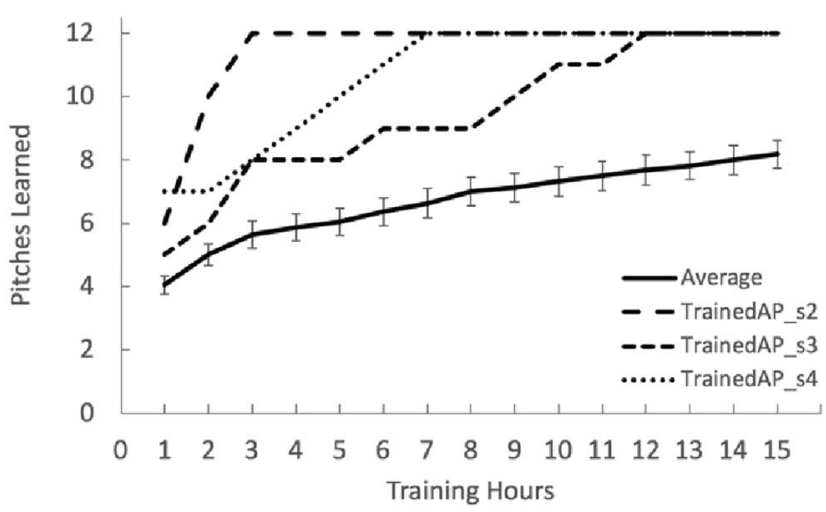

(c) Experiment 3

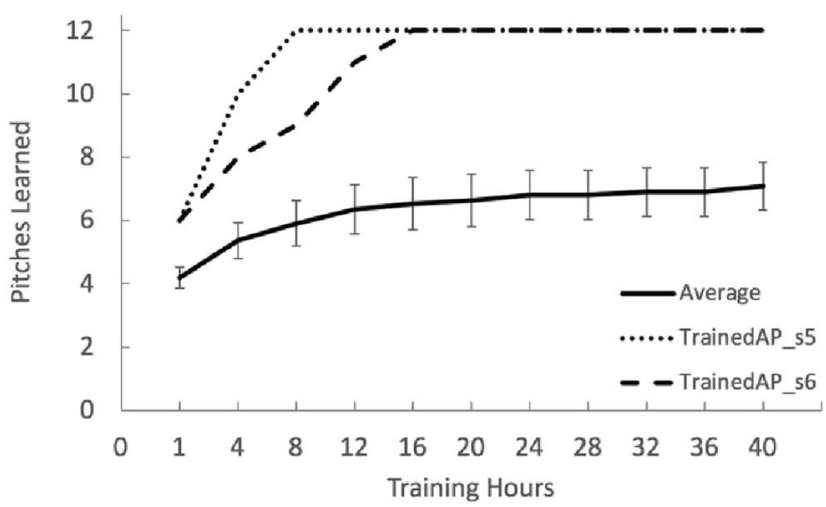

Fig. 3 The number of pitches learned in the course of training in the three experiments. Number of pitches learned was defined as the number of pitches included at the highest passed levels at 90\% accuracy with no feedback or scoring assistance in each individual. The solid line shows the average number of learned pitches across all individuals in each experiment. The other lines showed the course of training of the individuals who passed all levels of training in each experiment. TrainedAP s6 in Experiment 3 learned to name 12 pitches at $90 \%$ accuracy with a subset of training tones (in one octave and one timbre only at level 74) at the end of the $16^{\text {th }}$ hour of training, but she actually passed all levels of training (level 80) during the $18^{\text {th }}$ hour of training 
.771, with a smaller pitch naming error at posttest than pretest. No other main effect or interaction was observed, $p \mathrm{~s}>.15$, i.e., we did not observe any difference between the naming performance of tones in trained or untrained timbres and octaves $\left(\eta_{\mathrm{p}}{ }^{2}\right.$ of the main effect of Prepost was $.846, .638, .485$ for octave \& timbre trained, octave trained \& timbre untrained, octave untrained \& timbre trained conditions respectively). These suggest that the AP learning generalized to untrained octaves and timbres.

To check if the improvement sustained for a month, a oneway ANOVA was performed with Prepost (pretest / posttest / a month later) on pitch naming error with the trained tones ${ }^{5}$. It revealed a significant main effect of Prepost, $F(2,16)=18.8, p$ $<.001, \eta_{\mathrm{p}}{ }^{2}=.701$. Post-hoc Scheffé tests $(p<.05)$ showed that the pitch naming error reduced after training and remained similar a month later, $p>.73$ (Supplementary Table $1 ; \eta_{\mathrm{p}}{ }^{2}$ of the main effect of Prepost between the pretest and the immediate posttest was .840 and that between the pretest and the posttest a month later was .675). These suggest that the AP learning sustained for a month. Overall, the AP learning corresponded well with classic characteristics of perceptual learning in terms of performance enhancement, generalization and sustainability.

While we did not include any control group to control for the potential learning effect during repeated testing, the observed improvement during the test for generalization was unlikely explained by repeated testing per se. First, AP is notoriously famous for its difficulty to acquire in the literature. Second, the average progress per hour of training (where at least hundreds of trials were involved) was relatively slow (Fig. 3A). Third, the test-retest improvement was limited based on our pilot testing. Eleven participants repeated a similar AP task three times in the same session, with an optional break in between. The task was a pitch verification task, in which half of the trials showed the correct label of a presented tone, and the other half of the trials showed a label that was a semitone away from the correct label. Participants had to judge whether the label and the tone matched or not by keypress. Each task had 144 trials. Result showed that performance did not differ among the three attempts of the same task, $F(1,20)=1.94, p$ $=.17$ (mean d' $=.27, .42$ and .30 and $S D=.72, .84$ and .73 for the three attempts respectively), suggesting that the test-retest improvement in AP judgment was limited. Therefore simply performing the test for generalization twice can unlikely cause the substantial and sustained AP improvement after training.

\footnotetext{
$\overline{{ }^{5} \text { One participant }}$ did not participate in the testing one month later and was excluded from this analysis.
}

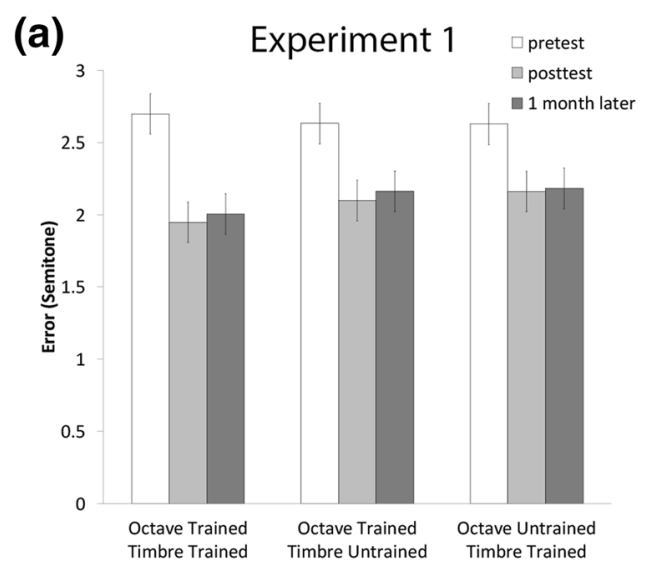

(b)
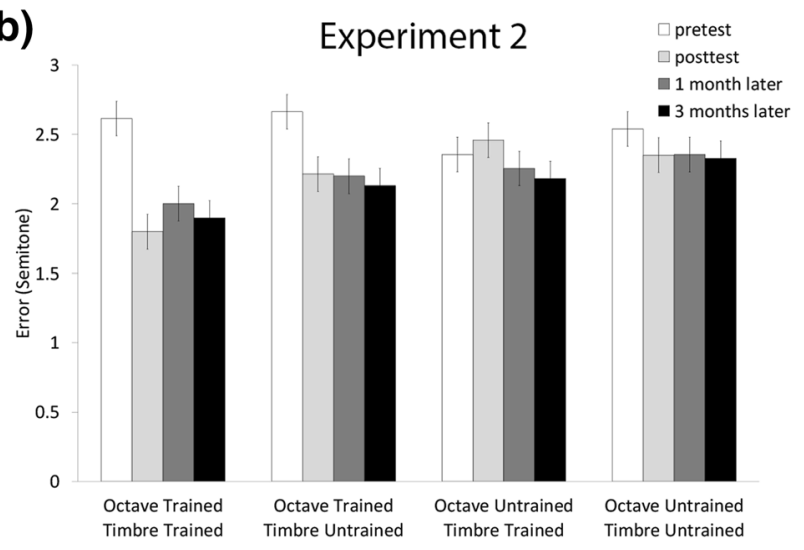

(c)
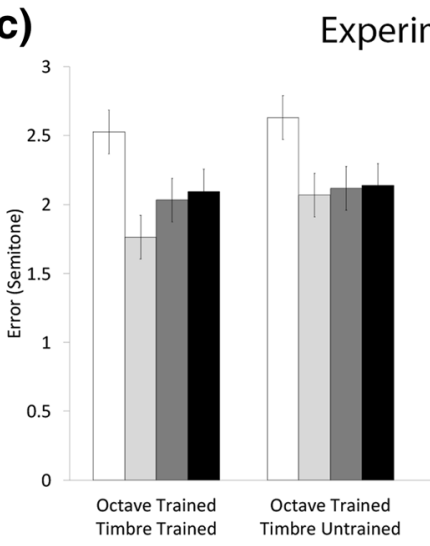

ment 3 Timbre Untrained

Fig. 4 Generalization of learning to different octaves and timbre in the three experiments. Pitch naming error was defined as the deviation in semitone from the correct response. Error bars represent 95\% CIs for the interaction effect between Prepost and Stimulus Type in Experiment 1 (A), for the interaction effect between Prepost, Octave and Timbre in Experiments 2 and 3 (B-C)

\section{Experiment 2}

Experiment 1 showed that it is feasible to acquire AP in adulthood, and the pattern of AP learning generally fits with that of perceptual learning. In Experiment 2, we aimed to replicate the feasibility of acquiring $\mathrm{AP}$ in adulthood and further characterize such AP learning. First, we tested the robustness of AP 
acquisition in adulthood by using a different set of training protocol, including a different set of training tones, training tasks, training duration and design (Table 1 and Fig. 1B). In particular, we used both a pitch verification task and a pitch naming task in the training. Compared with a naming task, the verification task had a reduced number of alternatives (ranged between 3 to 12 in a naming task) to 2 (yes / no) while keeping the perceptual demand of differentiating between tones that are one semitone apart. A smaller number of alternatives reduces the decisional demand, and thus makes the task easier (Y. K. Wong \& Wong, 2014). This may further break down AP learning into smaller steps such that AP can be improved more smoothly. Given that many participants were still slowly improving towards the end of the training in Experiment 1 (Supplementary Figure 2), the training time of Experiment 2 was also extended to 15 hours so as to allow time for improvement.

Second, Experiment 1 showed that including training tones in multiple octaves and timbres resulted in generalized AP learning to untrained tones. This result is consistent with the predictions based on the psychological space of training and testing (Y. K. Wong et al., 2011; see also Nosofsky, 1986, 1987; Palmeri \& Gauthier, 2004). In Experiment 2, we further tested the predictions of the psychological space by asking whether covering a more specific part of the psychological space, i.e., by using a smaller set tones in one octave and one timbre only, would lead to higher specificity in AP learning.

Third, we examined whether musicians benefit from the training more than non-musicians. The long-term musical training in musicians have fine-tuned the perceptual representations of tones in the auditory system (Pantev et al., 1998), which may in turn help one establish the association between the tones and the pitch names, i.e., learning AP.

\section{Methods}

\section{Participants}

Twenty-two participants, including ten musicians and twelve non-musicians who were native Cantonese speakers, were recruited at City University of Hong Kong and completed the training. The musicians included 4 males and 6 females and were 23.5 years old on average $(S D=3.34)$. They were trained in music for 6-14 years, with the major instrument being piano $(N=9)$ and guitar $(N=1)$. The non-musicians included 5 males and 7 females and were 21.8 years old on average ( $S D$ $=1.27$ ). They were not formally trained with music or had brief training that lasted for less than a year. Similar to Experiment 1, all participants produced an average pitchnaming error with the training tones that was larger than 1 semitone in the test for generalization before training (see below). Two additional musicians dropped out of the training soon after participating because they could not commit to the whole training. The sample size per group was determined by matching that of Experiment 1. All participants filled out a similar questionnaire on musical training background similar to that of Experiment 1. Participants received monetary compensation for the training and testing, with additional bonuses for passing each level of training.

\section{Materials \& Stimuli}

In Experiment 2, 72 tones in timbres of synthetic, piano and violin tones from octaves four to five were used. The tones were identical to that used in Experiment 1. An additional glissando clip in synthetic timbre, which was often perceived as an endless glissando travelling continuously from a high pitch to a low pitch (Deutsch, 1995; Deutsch, Hamaoui, \& Henthorn, 2007), was used to further interfere with any existing auditory memory of tones before AP testing (see below).

\section{Absolute pitch training}

The AP training followed that of Experiment 1 except for the following. The training lasted for 15 hours, and included only 12 synthetic tones from octave 4 . These tones were selected because they are in the middle range of the pitch height. There were 30 training levels, which were organized into ten 3-level parts with an increasing number of pitches (from 3 pitches in the first 3 levels to 12 pitches in the last 3 levels; Fig. 1B). Within each 3-level part, the first level involved a verification task, in which participants judged by key press whether the pitch of the presented tone matched with a pitch name shown on the screen (' $y$ ' for match and ' $n$ ' for mismatch) within $5 \mathrm{~s}$ (Fig. 2A). Trial-by-trial feedback was provided. The second level was a naming task as in the same level in Experiment 1 with trial-by-trial feedback (Fig. 2A). The third level was a similar naming task without trial-by-trial feedback.

While participants generally stayed at the same level if they did not pass it, for this third level, upon failure in three consecutive attempts, they would be moved back to the second level. This allowed participants to re-learn the materials with the assistance of trial-by-trial feedback in case they were not ready for the no-feedback training. The number of trials increased from 12 trials per level to 30 trials per level gradually, with two trials added every time a new pitch was introduced. This allowed the blocks of trials to better represent and capture the increasing number of pitches included in the training set.

Similar to Experiment 1, the no-feedback levels were designed to serve as mini milestones for participants' AP performance at $90 \%$ accuracy. To further destroy any existing auditory memory trace of tones before testing, a 15 s glissando clip was played after listening to the sample tones and before the start of the no-feedback levels (Figure 2A). This should have effectively interfered with any auditory memory trace of tones since a 15-s latency with ineffective rehearsal (because of 
interfering tasks or stimuli) has been shown to result in very low accuracy during recall $(10 \%$ or lower; Peterson \& Peterson, 1959; see also the discussion in Takeuchi \& Hulse, 1993; and Wengenroth et al., 2013). This further minimized the possibility that participants performed the no-feedback levels with the assistance of external reference tones.

\section{Test for generalization}

The test for generalization was performed before the training, within three days after it, a month later and three months later to examine how well the pitch-naming abilities generalized to untrained octaves and timbres. Seventy-two tones were used. The tones were either in octaves 4 to 5 , in which octave 4 was trained and octave 5 was untrained (Table 1). Also, the tones were in one of three timbres, with synthetic tones as a trained timbre, and piano and violin tones as untrained timbres. The test was administered with four conditions, either with trained octave and timbre, trained octave and untrained timbre, untrained octave and trained timbre, or untrained octave and timbre.

A similar pitch-naming task was used as in Experiment 1 (Fig. 2B). There were 144 trials presented in randomized order, with each tone presented twice. Ten practice trials were provided with feedback before testing. A 15 s glissando tone was presented after the practice and before the testing to minimize any auditory working memory of tones that were previously heard (Peterson \& Peterson, 1959; Takeuchi \& Hulse, 1993; Wengenroth et al., 2013). Pitch-naming error, i.e., the average error in semitone of participants' response in comparison with the correct response, was used as the dependent measure. The average percentage of no-response trials in the pretest, posttest, testing after one month and testing after three months was $.085 \%, .024 \%, .015 \%$ and $.005 \%$ respectively.

\section{Results and Discussion}

\section{Learning of AP}

In general, we replicated the feasibility of acquiring AP in adulthood. Participants made substantial progress in learning to name pitches. At the end of training, the musicians and nonmusicians were able to name on average 9.4 (range $=7-12$; SD $=2.12$ ) and 7.2 pitches (range $=5-9 ; \mathrm{SD}=1.34$ ) at $90 \%$ accuracy respectively (Fig. 3B; Supplementary Figure 3). A one-way ANOVA with Group (musician / non-musician) on the number of learned pitches by the end of training revealed a significant main effect of Group, $F(1,20)=9.06, p=.007, \eta_{\mathrm{p}}{ }^{2}$ $=.312$. This shows that musicians had a larger progress in learning AP than non-musicians under the same training regime.

Importantly, three out of the twenty-two participants passed all levels of training, meaning that they were able to name all of the twelve pitches at $90 \%$ accuracy without externally provided reference tones, i.e., they have acquired $\mathrm{AP}$ with performance comparable to that of real-world 'AP possessors' (for definition see Methods in Experiment 1). These suggest that $\mathrm{AP}$ acquisition in adulthood is not a result of a certain specific type of training protocol used in Experiment 1, but can be generally observed with different perceptual learning paradigms.

\section{Generalization \& sustainability of AP learning}

Pitch-naming performance improved and the improvement was larger for the trained than untrained tones (Fig. 4B). A 2 x 2 × 2 × 2 ANOVA with Group (musician / non-musician), Prepost (pretest / posttest), Octave (trained / untrained) and Timbre (trained / untrained) as factors on pitch naming error revealed a significant main effect of Prepost, $F(1,20)=25.6, p$ $<.001, \eta_{\mathrm{p}}{ }^{2}=.561$, as the pitch naming error reduced after training. There were significant interactions between Prepost and Octave, $F(1,20)=11.4, p=.003, \eta_{\mathrm{p}}{ }^{2}=.363$, and between Prepost, Octave and Timbre, $F(1,20)=13.8, p=.001, \eta_{\mathrm{p}}{ }^{2}=$ .408 . Post-hoc Scheffé test $(p<.05)$ showed that the precision of pitch naming was higher for tones with the trained octave and timbre than other tones only after training (Supplementary Table 2). Since the AP training in Experiment 2 only covered training tones from a single octave and timbre, the results are consistent with the hypothesis that the degree of learning specificity and generalization depends on the psychological multidimensional space of the training and testing stimuli (Nosofsky, 1986, 1987; Palmeri \& Gauthier, 2004; Y. K. Wong et al., 2011).

The results also suggest that the observed improvement during the tests for generalization cannot be explained by repeated testing per se. Specifically, in each test for generalization, each trained and untrained tone was presented with an equal number of trials. If the observed AP learning during the tests for generalization was caused by repeated testing per se, the AP improvement should be equivalent for the trained and untrained conditions. The larger improvement for the trained tones than untrained tones did not support this prediction.

Concerning the AP learning between musicians and nonmusicians, the interaction between Prepost and Group in the same ANOVA was significant, $F(1,20)=4.87, p=.039, \eta_{\mathrm{p}}{ }^{2}=$ .196 , in which both groups showed better performance at posttest than pretest, with a larger improvement in musicians $\left(M_{\text {pretest }}=2.39, S D=.64 ; M_{\text {posttest }}=1.88, S D=.76 ; \eta_{\mathrm{p}}{ }^{2}\right.$ for the main effect of Prepost $=.618)$ than non-musicians $\left(M_{\text {pretest }}=\right.$ 2.67, $S D=.36 ; M_{\text {posttest }}=2.47, S D=.53 ; \eta_{\mathrm{p}}{ }^{2}$ for the main effect of Prepost $=.470$ ). The main effect of Timbre was significant, $F(1,20)=8.93, p=.007, \eta_{\mathrm{p}}^{2}=.309$, which interacted with Group, $F(1,20)=5.29, p=.032, \eta_{\mathrm{p}}{ }^{2}=.209$. Post-hoc Scheffé test $(p<.05)$ showed that non-musicians performed better with the trained timbre than the untrained 
timbre, while musicians performed similarly with the two timbres (Supplementary Table 3). The four-way interaction did not reach significance $(F<1)$.

In sum, musicians learned to name more pitches than nonmusicians during the training, and showed a larger improvement than novices in the precision of pitch naming during the test for generalization. These suggest that previous exposure to and training with musical tones may facilitate AP acquisition. However, there are a few things to note. First, we recruited real-world musicians and non-musicians. Without manipulating musical experience, we could not exclude the possibility that pre-existing differences in the two groups might explain the group difference in AP learning. For example, musicians might simply be more motivated to learn AP than non-musicians because AP is musically relevant. Second, numerous studies have reported that musical training improves cognitive abilities, including verbal memory (Ho, Cheung, \& Chan, 2003), spatial-temporal reasoning (Rauscher et al., 1997), and IQ (Schellenberg, 2004). Therefore it is also possible that the more efficient learning of AP in musicians might have stemmed from their improved cognition. Third, musicians may have more likely practiced the newly acquired AP abilities between the pretest and posttest period. Fourth, quantifying musical experience as a continuous variable instead of a dichotomous one, e.g., either musicians or non-musicians as in the current study, might allow better understanding of the relationship between musical training and AP development (Daly \& Hall, 2018; MacCallum, Zhang, Preacher, \& Rucker, 2002). Future studies should clarify the factors underlying the AP learning advantage in musicians.

Finally, to check if the improvement sustained, a twoway ANOVA with Group (musician / non-musician) $\mathrm{x}$ Prepost (pretest / posttest / 1 month later / 3 months later) was performed on pitch naming error with the trained tones only ${ }^{6}$. It revealed a significant main effect of Prepost, $F(3,54)=17.4, p<.001, \eta_{\mathrm{p}}{ }^{2}=.492$. Post-hoc Scheffé tests $(p<.05)$ showed that pitch naming error was smaller at posttest than pretest, and stayed similar one and three months later (Supplementary Table $4 ; \eta_{\mathrm{p}}{ }^{2}$ of the main effect of Prepost between the pretest and each of the subsequent tests was $.614, .636$ and .554 for the posttest, 1 month later and 3 months later respectively), suggesting that the AP improvement sustained for at least three months. There was a trend that musicians performed better than non-musicians in general $(p=.09)$, but this effect did not interact with Prepost $(F<1)$. Overall, we replicated the perceptual learning nature of AP learning in adulthood in terms of performance enhancement and sustained improvement for at least three months.

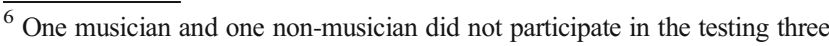
months later and were excluded from this analysis.
}

\section{Experiment 3}

Experiment 2 replicated the findings of Experiment 1 that AP can be learned in adulthood through laboratory perceptual training. AP training would be more relevant to musicians and the general public if it can be done in the real-world. However, it creates many challenges for the AP training because many factors cannot be controlled outside of the laboratory, including noise and distraction, training duration of each session, frequency of training, devices used for the training (computers, mobile phones, tablets, etc.), the mode of presentation of the stimuli (e.g., earphones, loudspeakers, etc.), the mode of response (e.g., mouse clicking, finger tapping), etc. In Experiment 3, we further asked whether AP training in adulthood is feasible outside of the laboratory. Participants performed an online AP training anywhere with a stable Internet connection. They each finished 40 hours of training in their own pace within an eight-week period.

\section{Methods}

\section{Participants}

Eleven participants who were native Cantonese speakers were recruited at City University of Hong Kong and completed the training. They included 4 males and 7 females and were 20.1 years old on average $(S D=$ 0.83 ). Six of them have received musical training for 3 to 15 years, with the major instrument being piano $(N=3)$, violin $(N=2)$ and flute $(N=1)$. Five were non-musicians who were not formally trained with music or had brief training that lasted for less than a year. The Distorted Tunes Test for tone-deaf screening was added before training (available at https://www.nidcd. nih.gov/tunestest/test-your-sense-pitch) so as to ensure that none of the participants would encounter difficulty in acquiring AP simply because of deficits in perceiving pitch in general. All participants performed the task satisfactorily with no suspected deficit with detecting distorted tunes (accuracy ranged from $73.1 \%$ to $100 \%$, $M=88.2 \%, S D=0.088$ ).

All participants produced an average pitch-naming error with the training tones that was larger than 1 semitone in the test for generalization before training, except one additional participant whose pre-training pitch naming precision was well-within the range of real-world 'AP possessors' $(0.37$ semitones from correct responses), leaving the training unnecessary and was excluded from the training. One additional musician was excluded from data analyses because she showed highly inconsistent and uninterpretable training 
performance ${ }^{7}$. The sample size was determined to match with that of Experiments 1 and 2. All participants filled out a similar questionnaire on musical training background similar to that of Experiment 1. Participants received monetary compensation for the training and testing, with additional bonuses for passing each level of training.

\section{Materials \& Stimuli}

In Experiment 3, 120 tones were used, including those in timbres of synthetic and piano tones in octaves four to six, and those in timbres of violin and clarinet in octaves four to five. The synthetic, piano and violin tones were identical to those used in Experiments 1 and 2. The clarinet tones were downloaded from online sound library (http://newt.phys.unsw.edu.au/music/ clarinet/index.html). The deviation between the actual frequency of the recorded tones and the expected frequency for the equal-tempered scale (with A4 = $440 \mathrm{~Hz}$ ) ranged from $0.029 \%$ to $1.00 \%$, with a mean deviation of $.373 \%, \mathrm{SD}=.270$. These tones were edited in an identical way as the other tones ${ }^{8}$. The same glissando clip in Experiment 2 was also used in this experiment.

\section{Absolute pitch training}

This training was administered online such that participants could be trained anywhere with stable Internet connection. The training lasted for 40 hours. The training time of the participants was counted into the total training time in units of 15 minutes (i.e., 17 minutes training was taken as 15 minutes, and 14 minutes training as 0 minute). This encouraged participants to plan ahead the time that they could commit into the training before they started, which might help participants identify appropriate time and place (e.g., with stable Internet connection) for the training. To minimize any idling time, the

\footnotetext{
$\overline{7}$ The performance pattern of this particular participant was highly inconsistent and uninterpretable. First, most participants tended to progress and improve more slowly as the training proceeded. However, this particular participant showed an opposite pattern. In particular, the progress of this participant was very slow in the first half of the training when only a few pitches were included. However, her progress suddenly and dramatically improved in the second half of the study when most of the pitches were included in the training. Also, she showed a large standard deviation in the number of attempts required to pass the training levels $(S D=55.4)$, which was more than two times higher than that of the rest of the group, showing that her performance was highly unstable and deviated from the learning patterns of others. Since the training was performed in an uncontrolled environment (unlike the previous experiments that were performed in the laboratory), the highly deviant learning pattern could be caused by many uninteresting reasons that are irrelevant to AP learning. Therefore, we decided to exclude the data of this participant for further data analyses.

${ }^{8}$ The intensity of the clarinet tones were $76.0 \mathrm{~dB}$ on average. The peak intensity was $78.8 \mathrm{~dB}$ and the mean of the root-mean-squared (RMS) values of the tones was .127.
}

session for listening to sample tones before each level would be ended with 10 s of inactivity. The program would automatically $\log$ out if there was inactivity for 3 minutes. They were trained for an average of five hours per week and finished the training in about eight weeks.

The design followed that of Experiment 2 except for the following. The training included 80 training levels, which were organized into ten 8-level parts (Figure 1C). It included piano, violin and clarinet tones in octaves 4 and 5 (Table 1). Different timbres were used to further test whether AP learning is robust to different timbres of the training tones.

To promote better learning, a summary table of the participant's performance was provided at the end of each level. The table showed the accuracy for each pitch and listed out the wrong answers provided for each pitch (if any). This information enabled participants to better evaluate the type of errors they made. Also, when the training set included 5 pitches or more, an additional compulsory exercise was introduced after every 10 attempts of passing the levels. The exercise would include 20 trials that centered around the worst-performed pitch in the past 10 attempted levels. If more than one pitches shared an identical and worst performance, the worstperformed pitch would be determined by a random draw among them. The timbre and octave of the tones would follow the level right before this exercise. This was designed to further help participants focus on the pitches that needed most training.

\section{Test for generalization}

The test for generalization was administered similarly to Experiment 2 except the following. 72 tones in octaves 4 to 6 (C4 to B6) were used, in which octaves 4 and 5 were trained, and octave 6 untrained (Table 1). Two timbres were included, with piano tones as the trained timbre, and synthetic tones as the untrained timbre. In the pitch naming task (Figure 2B), the trials were blocked in four conditions, either with a trained or untrained octave crossed with a trained or untrained timbre. There were 192 trials in total, with 48 trials in each condition. The trials within each block were randomized. The average percentage of no-response trials in the pretest, posttest, testing after one month and testing after three months was $.065 \%$, $.033 \%, .015 \%$ and $.011 \%$ respectively.

\section{Results and Discussion}

\section{Learning of AP}

In general, the participants made substantial progress in learning to name pitches. At the end of training, they were able to 
name on average 7.4 pitches (range $=5-12 ; S D=2.46$ ) at 90\% accuracy (Fig. 3C; Supplementary Figure 4).

Importantly, two out of the eleven participants passed all levels of training, meaning that they were able to name all of the twelve pitches at $90 \%$ accuracy without any externally provided reference tones. In other words, we further replicated the finding that AP can be acquired in adulthood, and demonstrated that AP training is feasible outside of the laboratory.

\section{Generalization \& sustainability of AP learning}

The online AP training improved pitch-naming performance in the laboratory, and the improvement generalized well to the untrained octave and less so to the untrained timbre (Figure 4C). A $2 \times 2 \times 2$ ANOVA with Prepost (pretest / posttest), Octave (trained / untrained) and Timbre (trained / untrained) as factors on pitch naming error revealed a significant main effect of Prepost, $F(1,10)=15.0, p=.003, \eta_{\mathrm{p}}{ }^{2}=$ .599 , as pitch naming error reduced after training. The interaction between Prepost and Timbre was significant, $F(1,10)=$ $7.15, p=.023, \eta_{\mathrm{p}}^{2}=.417$. Post-hoc Scheffé tests $(p<.05)$ showed that the pitch naming error was similar for trained and untrained timbres before training. While the error of both types of timbres reduced at posttest, the error was smaller for trained timbres than untrained timbres after training (Supplementary Table 5). The main effect of Octave was significant, $F(1,10)=15.8, p=.003, \eta_{\mathrm{p}}{ }^{2}=.609$, as the pitch naming error was smaller for the untrained than trained octave in general. However, the interaction between Prepost and Octave did not reach significance $(F<1)$, meaning that we did not observe any difference in the degree of AP learning for trained and untrained octaves $\left(\eta_{\mathrm{p}}^{2}\right.$ for the main effect of Prepost was .643 and .500 for the trained and untrained octaves respectively). The three-way interaction did not reach significance $(F<1)^{9}$. In sum, AP learning generalized to untrained tones similar to findings of Experiment 1. The improvement for trained timbres was larger than that of untrained timbres, while the improvement for trained and untrained octaves was similar. This indicated that the generalization of learning was more complete for untrained octave than timbre. Alternatively, the lack of generalization from the trained to the untrained timbre might be caused by the use of synthetic tones as the untrained timbre, since synthetic tones were known to be relatively difficult to label even among 'AP possessors' (see review in Takeuchi \& Hulse, 1993).

For the sustainability of the improvement, a one-way ANOVA with Prepost (pretest / posttest / 1 month later / 3 months later) on pitch naming error with the trained tones

\footnotetext{
${ }^{9}$ Including the participant with inconsistent pattern of learning in the ANOVA analyses resulted in largely similar results, except that the interaction between Prepost and Timbre was no longer significant, $F(1,11)=2.41, p=.15$, i.e., the $\mathrm{AP}$ learning generalized to untrained timbres and octaves completely.
}

revealed a main effect of Prepost, $F(3,27)=10.6, p<.001$, $\eta_{\mathrm{p}}{ }^{2}=.541^{10}$. Post-hoc Scheffé tests $(p<.05)$ showed that pitch naming error reduced after training, and stayed similar at all subsequent posttests (Supplementary Table $6 ; \eta_{\mathrm{p}}{ }^{2}$ of the main effect of Prepost between the pretest and each of the subsequent tests was $.676, .518$ and .522 for the posttest, 1 month later and 3 months later respectively), suggesting that the improvement sustained for at least three months. Overall, the performance enhancement, generalization and sustainability correspond well with that of the perceptual learning literature (Fahle \& Poggio, 2002; Goldstone, 1998; Y. K. Wong et al., 2011).

\section{Overall Analyses of Experiment 1 to 3}

\section{Characterizing individual progress of AP learning}

Is AP learning dichotomous, i.e., can one either acquire AP, or be completely unable to improve this ability? Instead of a dichotomous distribution, we observed a continuous distribution of learning progress in this study (Fig. 5), with a considerable number of participants acquiring every step between 5 to 12 pitches. If our training could further extend in time, it is well possible that more participants might be able to learn all of the twelve pitches (e.g., one participant who learned 11 pitches and two participants who learned 10 pitches by the end of the training). In general, our results were more consistent with the continuous view of AP ability (Bermudez et al., 2009; Levitin \& Rogers, 2005) than the dichotomous view (Athos et al., 2007; W. D. Ward, 1999; Zatorre, 2003).

\section{Characterizing the trained AP}

Despite the continuous training progress across participants, it is still informative to further examine the participants who learned to name all 12 pitches. An obvious question concerns whether these successfully trained participants were simply 'AP possessors' before training. Two aspects of our data indicate that they were not. One is that they required more time to pass all of the training levels than one would expect if they were already 'AP possessors' before training. Specifically, the AP training should be highly straightforward for 'AP possessors', and thus passing each of the levels should be easy with one or two attempts. If so, they should be able to pass the training well within the first hour of training. However, they required 7.83 hours on average to pass the training $(S D=5.23$; range: 3 to 18 ; Fig. 3), which was unreasonably long if they were 'AP possessors'. Second, their pretest performance of AP naming for the training tones was low $\left(M_{\text {error }}=1.97\right.$

\footnotetext{
${ }^{10}$ One non-musician did not participate in the posttest one month later and was excluded from this analysis.
} 
10

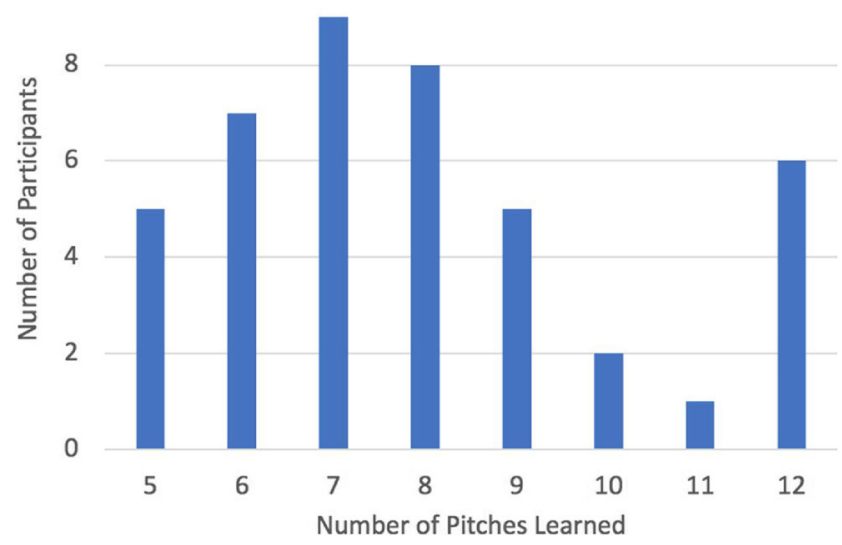

Fig. 5 The number of pitches learned by the end of training, with the participants ranked from low to high number of learned pitches

semitones; $S D_{\text {error }}=0.47$ ). This performance level, even considering the fact that the timbre and octave used may not be the most familiar to these individuals, would still be rarely considered typical of 'AP possessors' among the published studies that defined 'AP possessors' based on objective performance measures. Therefore, the pretest performance and training progress of these successfully trained participants did not support the idea that they were 'AP possessors' before training.

Next, was the music training background of the successfully trained participants, in which all of them were musicians, different from the other musicians? Our data indicated that the successfully trained participants were well within $3 \mathrm{SD}$ of the mean of the other musicians in various aspects of music training, suggesting that they fell within the distribution of other musicians in music training background. In terms of musical achievement, indicated by the highest passed level of ABRSM $^{11}$, the successfully trained participants were between -.936 and $1.63 \mathrm{SD}$ from the mean of the other musicians that participated in this study (mean $=$ Grade $6.46, S D=1.56$ ). For the age of onset of music training, the successfully trained participants were between -.374 and -1.10 SD from the mean of the other musicians (mean $=9.53$ years, $S D=4.09$ ). For the amount of music practice in the past 6 months, it was coded into 4 categories: Not At All (0 hour in the last 6 months), Rarely (less than 10 hours per half year), Sometimes (1-10 hours per month), and Frequent (1-10 hours per week or more). These 4 categories scored 1, 2, 3, 4 points respectively, which quantified the amount of music practice. The successfully trained participants ranged from -1.30 to $1.25 \mathrm{SD}$ from the mean of other musicians (mean $=2.53, \mathrm{SD}=1.18$ ). Overall, we did not see clear evidence that the successfully

\footnotetext{
${ }^{11}$ Four musicians were excluded from this analysis because they did not take part in any ABRSM before, therefore we did not have any data on their level of musical achievement. One participant passed the ATCL exam of the Trinity College London instead of the ABRSM, which was artificially taken as equivalent to Grade 9 of ABRSM for the current calculation.
}

trained participants had a different music training background from other musicians.

In the literature, it is well known that 'AP possessors' suffer from performance decrement with unfamiliar timbres and octaves (Levitin \& Rogers, 2005; Takeuchi \& Hulse, 1993; Y. K. Wong \& Wong, 2014). In our test for generalization, we included a large proportion of untrained tones with varied timbres and octaves, ranging from $66.7 \%$ to $75 \%$ of the trials, and the untrained tones were often intermixed with the trained tones. Was the trained AP ability also affected by the exposure to a large proportion of untrained tones? To address this question, we examined the AP performance of the trained tones during the posttest right after training for these six individuals. A one-way ANOVA with Prepost (pretest / posttest) on pitch naming error with the trained tones revealed a main effect of Prepost, $F(1,5)=16.4, p=.010, \eta_{\mathrm{p}}^{2}=.766$, indicating that the pitch naming error was smaller at posttest $\left(M_{\text {error }}=.925 \mathrm{semi}\right.$ tones; $\left.S D_{\text {error }}=0.447\right)$ than pretest $\left(M_{\text {error }}=1.83\right.$ semitones; $\left.S D_{\text {error }}=0.411\right)^{12}$. There was however a wide range of pitch naming errors across these trained individuals (range: $.375-$ 1.58 semitones), suggesting that the trained AP of some individuals were highly susceptible to the exposure to a large proportion of unfamiliar tones.

Finally, did the trained AP sustain for at least a month? A one-way ANOVA with Prepost (pretest / posttest / 1 month later) ${ }^{13}$ on pitch naming error with the trained tones revealed a main effect of Prepost, $F(2,10)=17.1, p<.001, \eta_{\mathrm{p}}{ }^{2}=.773$. Post-hoc LSD test $(p<.05)$ showed that the pitch naming error was smaller at posttest than pretest and remained similar a month later (For pretest, $M_{\text {error }}=1.83$ semitones; $S D_{\text {error }}=$ .411 ; For posttest, $M_{\text {error }}=.925$ semitones; $S D_{\text {error }}=.447$; For 1 month later, $M_{\text {error }}=.958$ semitones; $S D_{\text {error }}=0.501 ; \eta_{\mathrm{p}}{ }^{2}$ of the main effect of Prepost between the pretest and each of the subsequent tests was .766 and .848 for the posttest and 1 month later respectively), suggesting that the trained AP sustained for at least a month.

It is interesting to think about how the effortful and explicit AP learning as in the current study compares with the AP acquisition in real-world 'AP possessors', yet this question is difficult to address. Instead of going through deliberate and structured training, 'AP possessors' often report that their AP develops implicitly or naturally. Given the limited insights in the cognitive processes underlying our AP abilities (e.g., Chin, 2003; Takeuchi \& Hulse, 2003), it remains unclear what

\footnotetext{
${ }^{12}$ The results on the accuracy measure, as requested by a reviewer, were similar: A one-way ANOVA with Prepost (pretest / posttest / after 1 month) on pitch naming accuracy revealed a significant main effect, $F(2,10)=4.25, p$ $=.046, \eta_{\mathrm{p}}^{2}=.459$, indicating that the pitch naming accuracy improved at posttest and one month later compared with pretest (at pretest: $\mathrm{M}_{\text {accuracy }}=$ $.375, \mathrm{SD}_{\text {accuracy }}=.103$; at posttest: $\mathrm{M}_{\text {accuracy }}=.583, \mathrm{SD}_{\text {accuracy }}=.158$; after 1 month: $\mathrm{M}_{\text {accuracy }}=.540, \mathrm{SD}_{\text {accuracy }}=.210$ ). The range of accuracy at posttest was between .38 to .79 , and that after one month was between .29 to .80 .

${ }^{13}$ We did not include the posttest at three months later because this test was not included in Experiment 1.
} 
underlies the AP acquisition of the 'AP possessors'. Nevertheless, on top of how AP is shaped by experience (as discussed above), 'AP possessors' are also aware that their AP ability is constantly updated through implicit learning by environmental input, e.g., exposure to music classes and performance (West Marvin, VanderStel, \& Siu, 2019). There is not much in the findings of the current study that can speak to the similarities and differences between these explicit and implicit attempts of AP. More empirical work is needed to approach the question, including the comparison of neural mechanisms underlying trained participants and real-world AP possessors.

\section{Comparing different training paradigms}

The three training paradigms used in the three experiments differed in many ways, including the training stimuli, duration, the venue of training, and the design of the training progression (Fig. 1; Table 1). While it is difficult to pinpoint the contributions of specific training parameters, we can at least view these training paradigms as different packages and ask one question: Was any of the training paradigms more effective than the others in general? A one-way ANOVA with Experiment $(1 / 2$ /3) as a between-subject factor was performed on the number of learned pitches at the end of the training, with the number of learned pitches defined by the number of pitches included at the highest passed level without feedback. The main effect of Experiment did not reach significance $\left(F<1 ; \eta_{\mathrm{p}}{ }^{2}\right.$ of the main effect of Prepost was .852, .680, .593 for Experiment 1, 2 and 3 respectively), suggesting that the AP learning was not significantly different between training paradigms, despite of the many differences in training protocols, tasks, stimuli and durations in the three experiments.

Apart from the progress during training, we can also directly compare the degree of generalization in the three training paradigms. A $3 \times 2 \times 3$ ANOVA with Experiment $(1 / 2 / 3)$ as a between-subject factor, and Prepost (pretest / posttest) and Stimulus Type (octave \& timbre trained / octave trained \& timbre untrained / octave untrained \& timbre trained $)^{14}$ as two within-subject factors was performed on pitch naming error. Critically, the three-way interaction between Experiment, Prepost and Stimulus Type was significant, $F(4,82)=3.27, p=.015, \eta_{\mathrm{p}}{ }^{2}=.138$. Post-hoc Scheffé test $(p$ $<.05)$ showed that performance in all stimulus conditions were similar within each experiment at pretest (Supplementary Table 7). At posttest, however, pitch naming error was similar between the trained and untrained tones for Experiment 1 and 3, while the pitch naming error was smaller for the trained than untrained tones in Experiment 2. This confirmed that the generalization of learning to untrained tones was higher in Experiment 1 and 3 than in Experiment

\footnotetext{
$\overline{14}$ The octave-and-timbre-untrained condition did not exist in Experiment 1 and therefore it was not included in this analysis.
}

2. Experiment 1 and 3 included training tones in more octaves and timbres, and covered a larger part of the psychological space than that in Experiment 2. Hence this finding supports our hypothesis that the degree of generalization of AP learning depends on the overlap between the psychological space of training and testing tones (Y. K. Wong et al., 2011; see also Nosofsky, 1986, 1987; Palmeri \& Gauthier, 2004).

Could the differences in generalization of learning across experiments be alternatively explained by the different timbres used for the trained and untrained tones in different experiments? It should be noted that, as described in the Methods sections, the tones were truncated and only the middle part of the tones with more stable pitch were extracted into the recordings. As pointed out by a reviewer, the timbral information of the tones could have been partly removed or damaged. It is therefore possible that the timbres used in the current study would have affected AP learning differently if the tones were created differently to preserve the natural characteristics of the timbre. Still it is the case that both the number of timbres covered in traing and the combination of trained and test timbres differed across experiments. In future studies it would be informative to use stimuli that preserve the timbre information of the tones, and dissociate the effect of timbre coverage in training and the effect of timbre similarity between training and testing on learning generalization.

\section{General Discussion}

The observations that AP rarely occurs in the population and is difficult to learn in adulthood have led to the belief that AP is only possible for a few selected individuals - those with particular genetic makeup and musical training within a critical period in early childhood. Our study provides the first empirical evidence that pitch naming can improve considerably in adults in both laboratory and online settings. Importantly, $14 \%$ of the participants (6 out of 43) successfully acquired AP within the training period, with performance levels comparable to that of 'AP possessors' as defined in the literature. This proportion of training success is unprecedented in the literature of AP, suggesting that AP continues to be learnable in adulthood.

The pattern of AP learning is consistent with that of the perceptual learning literature. AP performance improved for all participants after training. In particular, the learning effects sustained for at least one to three months and generalized to untrained tones to an extent depending on the specificity of the training set. When the training set included tones from more octaves and timbres (e.g., Experiments 1 \& 3), AP learning generalized better to untrained tones, whereas the learning was more specific to the trained tones when the training targeted on a smaller set of tones (e.g., Experiment 2). The specificity of learning as a function of the psychological 
multidimensional space during training and testing is consistent with findings in perceptual learning studies (Fahle \& Poggio, 2002; Goldstone, 1998; Y. K. Wong et al., 2011). We did not observe any difference in the number of pitches learned in the three experiments that involved different training protocols, tasks, stimuli and durations. This suggests that AP learning in adulthood can be achieved with a wide range of training regimes.

\section{Did the training lead to 'genuine AP'?}

In this study, AP was empirically defined based on performance in a pitch-naming task. It is almost the only empirical measure used in the literature to distinguish between 'AP possessors' and others, based on which insights on absolute pitch processing have been obtained. Also, the criterion used for AP acquisition (90\% accuracy in naming all 12 pitches, with one semitone error regarded as incorrect) is more stringent than that used in the majority of empirical studies thus far (see Methods of Experiment 1). We therefore deem it appropriate to use this operational definition to assess AP acquisition.

One may question whether the participants may have used relative pitch strategies to facilitate their AP performance during training and testing, given that sample tones and feedback were provided for certain training trials, and practice trials during pre- and posttests. While it could be the case for Experiment 1, a $15 \mathrm{~s}$ glissando clip was introduced in Experiments 2 and 3 after listening to the sample tones during training, before the start of the no-feedback levels of the training, and after the feedback-provided practice trials during preand posttests. This has been shown to be effective in interfering with and minimizing the auditory memory trace left by any sample tones heard (Peterson \& Peterson, 1959; Wengenroth et al., 2013). If, even with the glissando clip, the sample tones still served effectively as externally provided reference, then the AP training and testing should essentially become relative pitch training and testing. This should have resulted in much better performance in our training and testing, which was not the case - the pretest performance was chance-like (Fig. 4) and the training progressed very slowly on average (Fig. 3).

Still, an alternative interpretation of the current finding is that the participants simply improved their accuracy in naming pitches through training, but were yet to acquire 'genuine AP'. With the relatively long time windows allowed for response ( $5 \mathrm{~s}$ for choosing one of twelve options by key pressing or mouse clicking), participant could have generated reference tones internally in a trial and used it for subsequent trials. In other words, participants could have learned to represent some of the pitches in an absolute manner while naming other pitches by comparing them to the internally generated references. While one can make such relative pitch comparisons more difficult by introducing a large pitch interval between adjacent trials, it was impossible in the current study given the pitches presented were generally selected from narrow ranges (within one to two octaves). If comparison with internal references is indeed what participants in general learned to do, then the ability acquired would be considered by some researchers as 'quasi-AP' (despite their accurate AP performance without relying on external reference tones), rather than the 'genuine AP' involving the representation of every pitch in an absolute manner (e.g., Levitin \& Rogers, 2005).

Regarding this alternative interpretation, two points are worth noting. First, the majority of empirical research has identified individuals as 'AP possessors' based on performance alone, without regarding to the underlying representation of pitch through which the high performance level is achieved. Our findings here would therefore be relevant and informative to the research field in general. Second, it is certainly interesting and important to ask whether those identified as 'AP possessors' in most empirical studies indeed represent every pitch absolutely. However, to date there has not been an agreed upon way to ascertain whether an individual perceives every pitch in an absolute manner or not. One could perhaps use fast responses to infer automatic pitch naming in an absolute manner. But the search for the threshold response time could be elusive - given that many factors would greatly affect response times, such as the stimulus set, the types of response (voice, key press, mouse click, etc.) and age - how fast is 'sufficiently fast' to represent 'automatic' pitch naming? Without an objective way to reveal the underlying representation of pitch of 'AP possessors', the assumption of 'genuine AP', as defined by representing every pitch absolutely, remains an untestable hypothesis.

Third, despite many differences in the methods and measurements, we compared the correct response time for a pitch naming task between the trained AP in the current study and the real-world 'AP possessors'. In the 6 participants who successfully acquired AP during training, their average correct response time during their last attempted level at which they passed the training was $2061.2 \mathrm{~ms}, S D=270.1$. In the papers that reported correct response time for a pitch naming task with realword 'AP possessors', the correct response time ranged from $1216 \mathrm{~ms}$ to $1662 \mathrm{~ms}$ (Carroll, 1975; Miyazaki, 1989, 1990; Takeuchi \& Hulse, 1993). It is important to note that all these papers explicitly requested participants to respond as quickly as possible, while we instructed our participants that speedy response was not important as long as it was made within the $5 \mathrm{~s}$ response time window. Therefore it is possible that difference in instruction alone would be sufficient to account for the observed difference in response time 
between the two groups. Also, factors related to stimuli (the octaves and timbre involved), response format (keyboard, mouse or keys on a piano) could have affected the results. Overall, it did seem likely that the response time of the successfully trained participants were not drastically different with those of the traditional 'AP possessors' documented in the literature. Future research with a larger sample of individuals with and without AP in the same study can examine if there are any qualitative and quantitative differences in terms of response time, accuracy, brain areas involved, etc. It will be then possible to investigate if the trained AP possessors recruit similar cognitive and neural mechanisms as the very best natural 'AP possessors'.

\section{Challenges to explaining AP occurrence with genes and the critical period}

Our finding of adults acquiring AP across all three experiments challenges the strong form of the genetic accounts of AP development that only a very small percentage of the population possesses the rare gene(s) that enables AP acquisition (e.g., Drayna, 2007). Within our training duration, the number of participants who acquired AP was likely a lower bound of the percentage of training success in adulthood. If our training could further extend in time, it is well possible that more participants might be able to acquire AP. The current results suggest that any relevant genetic dispositions that enable AP acquisition should be a lot more prevalent in the population than previously assumed (Baharloo et al., 1998; Drayna, 2007). However, it is still possible that genetic factors may explain individual differences in AP acquisition.

Our finding also challenges the theoretical proposal that the critical period constrains the development of high-level cognitive abilities. This converges with findings that high-level visual and musical abilities continue to develop in adulthood (Daw, 1998; Trainor, 2005). This is also consistent with findings in language development (Flege et al., 1995; Singleton, 2001) and synesthesia (Bor et al., 2015) that acquisition of these abilities beyond the critical period is possible. The critical period may instead mainly apply to the development of basic structures and functions, such as the anatomical development of the visual system based on the environmental inputs (Hubel \& Wiesel, 1970; Simons \& Land, 1987; Wiesel \& Hubel, 1963; Zhang et al., 2002; but see Hooks \& Chen 2007).

Nonetheless, there could still be a 'sensitive period' in early childhood for AP development. For example, the same training experience may help young children develop AP to a larger extent than that in adults (Hooks \& Chen, 2007; Sengpiel, 2007). Future research may compare the effectiveness of AP training in young children and adults to address this question.
The participants in the current study were native speakers of Cantonese, which is a tonal language. In tonal languages, words that are different only in tones (pitch heights or contour) can have entirely different semantic meanings. For example, in Cantonese, the word 'ma' means 'mother' in tone one, 'grandmother' in tone four, 'horse' in tone five, and 'to scold' in tone six. It has been proposed that tonal language speakers learn to associate words with tonal templates that are absolute, precise and stable during the critical period of language development, which could later facilitate the development of AP (Deutsch et al., 2009; Deutsch, Henthorn, \& Dolson, 2004; Deutsch, Henthorn, Marvin, \& Xu, 2006). However, this hypothesis also assumes a speech-based critical period of AP acquisition for tonal language speakers:

"On this line of reasoning, it was hypothesized that in cultures where tone languages are spoken, infants generally acquire AP for the tones of their language during the critical period in which they acquire other features of their native language (Deutsch, 2002). When they reach the age at which they can begin musical training, they acquire AP for musical tones in the same way as they would acquire the tones of a second tone language (see, for example, Wayland and Guion, 2004). For such individuals, therefore, the acquisition of AP for musical tones should also be subject to a critical period." (p.2399, Deutsch et al., 2009)

Therefore the hypothesis predicts that the acquisition of AP is impossible in adulthood even for tonal language speakers. Our findings challenge the existence of such a speech-based critical period of AP acquisition. Alternatively, it is still possible that learning tonal language as the first language may facilitate AP learning in adulthood as in the current study, though such facilitation would be theoretically irrelevant to the concept of critical period since it has been way passed. Future studies may compare AP acquisition in tonal and nontonal language speakers with various factors (musical background and experience, social-economic status, intelligence, etc.) controlled to understand the contribution of tonal language background on AP development.

\section{Understanding AP acquisition from the learning perspective}

Integrating findings in the AP literature and the current study, we propose that the genesis of AP is better understood from a learning perspective. First, AP continues to be learnable from childhood (Crozier, 1997; Miyazaki \& Ogawa, 2006; Sakakibara, 2014) to adulthood (the current study), from single tones (the current study; Brady, 1970; Meyer, 1899) to complex melodies and songs (Halpern, 1989; Levitin, 1994; Schellenberg \& Trehub, 2003), and from musicians, non- 
musicians to the general public (Experiment 2 of the current study; Levitin, 1994; Schellenberg \& Trehub, 2003). This suggests that AP is learnable in a wide range of ages, stimuli and individuals. Second, even for the 'AP possessors' that have stably acquired AP, they can still learn to fine-tune their AP representations based on recent environmental input (e.g., detuned music; Hedger et al., 2013), suggesting that their AP representations remains actively alterable instead of being 'hard-wired'. Third, the learning perspective is consistent with the substantial evidence showing that AP is shaped by experience. For example, AP performance is better with the timbre of one's own instrument (Takeuchi \& Hulse, 1993), the highly exposed pitch like 'A4' (Levitin \& Rogers, 2005; Takeuchi \& Hulse, 1993), the multisensory testing context similar to one's musical training (Y. K. Wong \& Wong, 2014). Overall, AP is learnable, involves alterable representations after acquisition and displays experience-dependent characteristics, supporting that the genesis of AP can be understood from a learning perspective.

The learning perspective also provides a parsimonious account of the differential manifestations of AP ability for different individuals. Various subtypes of AP have been introduced to explain AP ability that is well above chance but not comparable with that of the so-called 'genuine AP possessors'. For example, 'pseudo AP', 'quasi-AP', 'implicit AP', 'latent AP' and 'residual AP' have been used to refer to non-discarded AP information during development, unexpressed potential to develop AP limited by the critical period, incomplete forms of AP, etc. (Deutsch, 2013; Levitin \& Rogers, 2005; Schellenberg \& Trehub, 2003; Takeuchi \& Hulse, 1993; W. D. Ward, 1999). These categorizations are established based on the assumption of a critical period in AP development. Given our challenge to the existence of the critical period on AP, it is doubtful how useful these categorizations are in enhancing our understanding of AP. Instead of being qualitatively different forms of AP, they may simply reflect different degrees and types of experience, and/or the attention and motivation in learning to process pitch information in an absolute manner. For instance, the ability to label the 'A4' tone only (referred as 'quasi-AP' or the 'absolute $\mathrm{A}^{\prime}$ ) can be best explained by their habitual use of A4 as the tuning tone (Levitin \& Rogers, 2005). The 'imperfect' or 'incomplete' AP performance may simply reflect various degree of AP learning, such as those demonstrated by the majority of the participants in this study (Figures 3 and 5; Supplementary Figure 1-3). Research on whether there are qualitative or quantitative differences between different types of AP would be a fruitful future direction.

From the learning perspective, we should all have the potential to acquire AP regardless of age or prior musical training. While successful learning can be affected by many factors such as motivation and persistence (Vansteenkiste, Simons, Lens, Sheldon, \& Deci, 2004), the difficulty to acquire AP, in terms of the slow improvement and relatively low success rate, is in clear contrast with the relative ease of other types of learning. For example, children can learn to name familiar objects in new languages fairly easily (Gathercole \& Baddeley, 1990), and adults can learn to name tens of novel objects with nonwords in several hours (A. C.-N. Wong et al., 2009; Y. K. Wong et al., 2011). What makes naming the twelve pitches so difficult to learn?

To speculate, a possible reason concerns the interference from speech. Speech contains rich and fine-grained tonal information, such as pitch levels and pitch contours, that conveys different meanings and emotions (Saffran \& Griepentrog, 2001). With years of experience in perceiving speech, tones and speech words may have formed intricate many-to-many mappings with a range of tones, or tonal patterns that are associated with different meanings and contexts. These experience-dependent mappings could be specific to each individual. It is possible that retrieving verbal labels of tones during a pitch-naming task activates the many-to-many mappings between tones and speech words, and that interferes with one's judgment of the pitch name of the tone. This proposal is consistent with the observation that musicians performed worse with tones presented with human voice than other timbres in a pitch labelling task (Vanzella \& Schellenberg, 2010). The individual difference in speech interference could drive the individual variability in AP performance and acquisition. Future work can further explore this possibility in explaining pitch-naming performance in the general public.

\section{Conclusion}

Overall, the current study shows that AP continues to be learnable in adulthood. The results challenge the influential theory that AP is only possible for few individuals with particular genes and training within the critical period. Instead, the role of learning should be given a larger emphasis. The findings also form a good basis for future research on identifying, if any, aspects of AP more trainable in adulthood and aspects that are potentially exclusive for the few 'AP possessors' observed in the real-world.

Acknowledgements The authors declare no conflict of interest. We thank Gabriel Chan Pak Hong and Michael Lai Wei Chun for their help in data collection, Mandy Chu Yan Ting for the technical support, Helen Wong Hoi Shan for her help in violin tone production, and Patrick Bermudez for providing the synthetic tones. 
Open Practices Statement The data and materials for all experiments are available at https://osf.io/tkrgb/files. None of the experiments was preregistered.

Authors Contributions Y. Wong and A. Wong developed the study concept and designed the study. Y. Wong collected the data. Y. Wong, K. Lui and K. Yip analyzed the data. Y. Wong, K. Lui and A. Wong drafted the manuscript. All authors approved the final version of the manuscript for submission.

\section{References}

Ahissar, M., \& Hochstein, S. (2004). The reverse hierarchy theory of visual perceptual learning. Trends in Cognitive Sciences, 8(10), 457-464. https://doi.org/10.1016/j.tics.2004.08.011

Athos, E. A., Levinson, B., Kistler, A., Zemansky, J., Bostrom, A., Freimer, N., \& Gitschier, J. (2007). Dichotomy and perceptual distortions in absolute pitch ability. Proceedings of the National Academy of Sciences of the United States of America, 104(37), 14795-14800. https://doi.org/10.1073/pnas.0703868104

Bachem, A. (1940). The Genesis of Absolute Pitch. The Journal of the Acoustical Society of America, 11, 434: https://doi.org/10.1121/1. 1916056

Baharloo, S., Johnston, P. A, Service, S. K., Gitschier, J., \& Freimer, N. B. (1998). Absolute pitch: an approach for identification of genetic and nongenetic components. American Journal of Human Genetics, 62(2), 224-231. https://doi.org/10.1086/301704

Baharloo, S., Serviceko, S. K., Risch, N., Gitschier, J., \& Freimer, N. B. (2000). Familial Aggregation of Absolute Pitch. The American Journal of Human Genetics, 67(3), 755-758. https://doi.org/10. $1086 / 303057$

Banai, K., \& Lavner, Y. (2014). The effects of training length on the perceptual learning of time-compressed speech and its generalization. The Journal of the Acoustical Society of America, 136(4), 1908-1917. https://doi.org/10.1121/1.4895684

Bermudez, P., Lerch, J. P., Evans, A. C., \& Zatorre, R. J. (2009). Neuroanatomical correlates of musicianship as revealed by cortical thickness and voxel-based morphometry. Cerebral Cortex, 19, 1583-1596.

Bermudez, P., \& Zatorre, R. J. (2005). Conditional associative memory for musical stimuli in nonmusicians: implications for absolute pitch. The Journal of Neuroscience : The Official Journal of the Society for Neuroscience, 25(34), 7718-7723. https://doi.org/10.1523/ JNEUROSCI.1560-05.2005

Bermudez, P., \& Zatorre, R. J. (2009). A Distribution of Absolute Pitch Ability as Revealed by Computerized Testing. Music Perception, 27(2), 89-101. https://doi.org/10.1525/mp.2009.27.2.89

Bor, D., Rothen, N., Schwartzman, D. J., Clayton, S., \& Seth, A. K. (2015). Adults Can Be Trained to Acquire Synesthetic Experiences. Scientific Reports, 4(1), 7089. https://doi.org/10. 1038/srep07089

Brady, P. T. (1970). Fixed-Scale Mechanism of Absolute Pitch. The Journal of the Acoustical Society of America, 48(4B), 883-887. https://doi.org/10.1121/1.1912227

Brainard, D. H. (1997). The psychophysics toolbox. Spatial Vision, 10, 433-436.

Brancucci, A., Dipinto, R., Mosesso, I., \& Tommasi, L. (2009). Vowel identity between note labels confuses pitch identification in nonabsolute pitch possessors. PloS One, 4(7), e6327. https://doi.org/ 10.1371/journal.pone.0006327

Carroll, J. B. (1975). Speed and accuracy of absolute pitch judgments: some latter-day results. ETS Research Bulletin Series, 1975(2), 0i71. https://doi.org/10.1002/j.2333-8504.1975.tb01075.x

Chin, C. S. (2003). The development of absolute pitch: a theory concerning the roles of music training at an early developmental age and individual cognitive style. Psychology of Music, 31(2), 155-171. https://doi.org/10.1177/0305735603031002292

Chung, S. T. L., \& Truong, S. R. (2013). Learning to identify crowded letters: Does the learning depend on the frequency of training? Vision Research, 77, 41-50. https://doi.org/10.1016/j.visres.2012. 11.009

Crozier, J. B. (1997). Absolute Pitch: Practice Makes Perfect, the Earlier the Better. Psychology of Music. https://doi.org/10.1177/ 0305735697252002

Cuddy, L. L. (1968). Practice effects in the absolute judgment of pitch. The Journal of the Acoustical Society of America, 43, 1069-1076. https://doi.org/10.1121/1.1910941

Cuddy, L. L. (1970). Training the absolute identification of pitch. Perception \& Psychophysics, 8(5A), 265-269.

Daly, H. R., \& Hall, M. D. (2018). Not all musicians are created equal: Statistical concerns regarding the categorization of participants. Psychomusicology: Music, Mind, and Brain, 28(2), 117-126. https://doi.org/10.1037/pmu0000213

Daw, N. W. (1998). Critical Periods and Amblyopia. Archives of Ophthalmology, 116(4), 502. https://doi.org/10.1001/archopht.116. 4.502

Deutsch, D. (1995). Musical illusions and paradoxes. La Jolla, CA: Philomel Records. (compact disc).

Deutsch, D. (2002). The puzzle of absolute pitch. Current Directions in Psychological Science, 11(6), 200-204.

Deutsch, D. (2013). Absolute Pitch. In The Psychology of Music (pp. 141-182). https://doi.org/10.1016/B978-0-12-381460-9.00005-5

Deutsch, D., Dooley, K., Henthorn, T., \& Head, B. (2009). Absolute pitch among students in an American music conservatory: Association with tone language fluency. The Journal of the Acoustical Society of America, 125(4), 2398-2403. https://doi.org/10.1121/1.3081389

Deutsch, D., Hamaoui, K., \& Henthorn, T. (2007). The glissando illusion and handedness. Neuropsychologia, 45, 2981-2988. https://doi.org/ 10.1016/j.neuropsychologia.2007.05.015

Deutsch, D., Henthorn, T., \& Dolson, M. (2004). Absolute Pitch, Speech, and Tone Language: Some Experiments and a Proposed Framework. Music Perception, 21(3), 339-356. https://doi.org/10. 1525/mp.2004.21.3.339

Deutsch, D., Henthorn, T., Marvin, E., \& Xu, H. (2006). Absolute pitch among American and Chinese conservatory students: Prevalence differences, and evidence for a speech-related critical period. The Journal of the Acoustical Society of America, 119(2), 719. https:// doi.org/10.1121/1.2151799

Drayna, D. (2007). Absolute pitch: a special group of ears. Proceedings of the National Academy of Sciences of the United States of America, 104(37), 14549-14550. https://doi.org/10.1073/pnas.0707287104

Fahle, M., \& Poggio, T. (2002). Perceptual learning. Massachusetts: MIT Press.

Fiorentini, A., \& Berardi, N. (1980). Perceptual learning specific for orientation and spatial frequency. Nature, 287(5777), 43-44. Retrieved from https://doi.org/10.1038/287043a0

Flege, J. E., Munro, M. J., \& MacKay, I. R. A. (1995). Factors affecting strength of perceived foreign accent in a second language. The Journal of the Acoustical Society of America, 97(5), 3125-3134. https://doi.org/10.1121/1.413041

Fujioka, T., Ross, B., Kakigi, R., Pantev, C., \& Trainor, L. J. (2006). One year of musical training affects development of auditory corticalevoked fields in young children. Brain, 129, 2593-2608. https:// doi.org/10.1093/brain/awl247

Gathercole, S. E., \& Baddeley, D. A. (1990). Phonological memory deficits in language-disordered children: Is there a causal connection? Journal of Memory and Language, 29, 336-360.

Gervain, J., Vines, B. W., Chen, L. M., Seo, R. J., Hensch, T. K., Werker, J. F., \& Young, A. H. (2013). Valproate reopens critical-period learning of absolute pitch. Frontiers in Systems Neuroscience, 7, 102. https://doi.org/10.3389/fnsys.2013.00102 
Goldstone, R. (1998). Perceptual learning. Annual Review of Psychology, 49, 585-612.

Gregersen, P. K., Kowalsky, E., Kohn, N., \& Marvin, E. W. (1999). Absolute Pitch: Prevalence, Ethnic Variation, and Estimation of the Genetic Component. The American Journal of Human Genetics, 65(3), 911-913. https://doi.org/10.1086/302541

Gregersen, P. K., Kowalsky, E., Kohn, N., \& Marvin, E. W. (2001). Early childhood music education and predisposition to absolute pitch: Teasing apart genes and environment. American Journal of Medical Genetics, 98(3), 280-282. https://doi.org/10.1002/10968628(20010122)98:3<280::AID-AJMG1083>3.0.CO;2-6

Gregersen, P. K., Kowalsky, E., Lee, A., Baron-Cohen, S., Fisher, S. E., Asher, J. E., ... Li, W. (2013). Absolute pitch exhibits phenotypic and genetic overlap with synesthesia. Human Molecular Genetics, 22(10), 2097-2104. https://doi.org/10.1093/hmg/ddt059

Halpern, A. R. (1989). Memory for the absolute pitch of familiar songs. Memory \& Cognition, 17, 572-581.

Hantz, E. C., Crummer, G. C., Wayman, J. W., Walton, J. P., \& Frisina, R. D. (1992). Effects of musical training and absolute pitch on the neural processing of melodic intervals: A P3 event-related potential study. Music Perception, 10(1), 25-42. Retrieved from http://www. jstor.org/stable/10.2307/40285536

Hartman, E. B. (1954). The influence of practice and pitch-distance between tones on the absolute identification of pitch. The American Journal of Psychology, 67(1), 1-14. https://doi.org/10.2307/ 1418067

Hedger, S. C., Heald, S. L. M., \& Nusbaum, H. C. (2013). Absolute pitch may not be so absolute. Psychological Science, 24(8), 1496-1502. https://doi.org/10.1177/0956797612473310

Hirose, H., Kubota, M., Kimura, I., Ohsawa, M., Yumoto, M., \& Sakakihara, Y. (2002). People with absolute pitch process tones with producing P300. Neuroscience Letters, 330(3), 247-250. https://doi. org/10.1016/S0304-3940(02)00812-1

Ho, Y. C., Cheung, M. C., \& Chan, A. S. (2003). Music training improves verbal but not visual memory: Cross-sectional and longitudinal explorations in children. Neuropsychology. https://oi.org/10.1037/ 0894-4105.17.3.439

Hooks, B. M., \& Chen, C. (2007). Critical periods in the visual system: changing views for a model of experience-dependent plasticity. Neuron, 56(2), 312-326. https://doi.org/10.1016/j.neuron.2007.10. 003

Hubel, D. H., \& Wiesel, T. N. (1970). The period of susceptibility to the physiological effects of unilateral eye closure in kittens. The Journal of Physiology, 206(2), 419-436. https://doi.org/10.1113/jphysiol. 1970.sp009022

Itoh, K., Suwazono, S., Arao, H., Miyazaki, K., \& Nakada, T. (2005). Electrophysiological correlates of absolute pitch and relative pitch. Cerebral Cortex (New York, N.Y. : 1991), 15(6), 760-769. https:// doi.org/10.1093/cercor/bhh177

Jäncke, L., Langer, N., \& Hänggi, J. (2012). Diminished Whole-brain but Enhanced Peri-sylvian Connectivity in Absolute Pitch Musicians. Journal of Cognitive Neuroscience, 24(6), 1447-1461.

Karni, A., \& Sagi, D. (1993). The time course of learning a visual skill. Nature, 365(6443), 250-252. Retrieved from https://doi.org/10. $1038 / 365250 \mathrm{a} 0$

Keenan, J. P., Thangaraj, V., Halpern, A. R., \& Schlaug, G. (2001). Absolute pitch and planum temporale. NeuroImage, 14(6), 14021408. https://doi.org/10.1006/nimg.2001.0925

Klein, M., Coles, M. G. H., \& Donchin, E. (1984). People with Absolute Pitch Process Tones Without Producing a P300. Science, 223(4642), 1306-1309. https://doi.org/10.1126/science.223.4642.1306

Knudsen, E. I. (2004). Sensitive Periods in the Development of the Brain and Behavior. Journal of Cognitive Neuroscience, 16(8), 14121425. https://doi.org/10.1162/0898929042304796

Kraus, N., \& Banai, K. (2007). Auditory-processing malleability: Focus on language and music. Current Directions in Psychological
Science, 16(2), 105-110. https://doi.org/10.1111/j.1467-8721.2007. 00485. $\mathrm{x}$

Lenhoff, H. M., Perales, O., \& Hickok, G. (2001). Absolute Pitch in Williams Syndrome. Music Perception, 18(4), 491-503. https:// doi.org/10.1525/mp.2001.18.4.491

Levitin, D. J. (1994). Absolute memory for musical pitch: evidence from the production of learned melodies. Perception \& Psychophysics, 56, 414-423. https://doi.org/10.3758/BF03206733

Levitin, D. J., \& Rogers, S. E. (2005). Absolute pitch: perception, coding, and controversies. Trends in Cognitive Sciences, 9(1), 26-33. https:// doi.org/10.1016/j.tics.2004.11.007

Loui, P., Li, H. C., Hohmann, A., \& Schlaug, G. (2011). Enhanced cortical connectivity in absolute pitch musicians: A model for local hyperconnectivity. Journal of Cognitive Neuroscience, 23(4), 1015-1026. https://doi.org/10.1162/jocn.2010.21500

Loui, P., Zamm, A., \& Schlaug, G. (2012). Enhanced functional networks in absolute pitch. NeuroImage, 63(2), 632-640. https://doi.org/10. 1016/j.neuroimage.2012.07.030

Lundin, R. W. (1963). Can perfect pitch be learned? Music Educators Journal, 49(5), 49-51.

MacCallum, R. C., Zhang, S., Preacher, K. J., \& Rucker, D. D. (2002). On the practice of dichotomization of quantitative variables. Psychological Methods, 7(1), 19-40. https://doi.org/10.1037/1082989X.7.1.19

Maurer, D., \& Mondloch, C. J. (2006). The infant as synesthete? In Processes of change in brain and cognitive development: Attention and performance XXI (pp. 449-471).

Meyer, M. (1899). Is the memory of absolute pitch capable of development by training? Psychological Review. https://doi.org/10.1037/ h0069034

Miyazaki, K. (1989). Absolute pitch identification: Effects of timbre and pitch region. Music Perception, 7, 1-14.

Miyazaki, K. (1990). The Speed of Musical Pitch Identification Possessors by Absolute-Pitch Possessors. Music Perception, 8(2), 177-188. https://doi.org/10.2307/40285495

Miyazaki, K., \& Ogawa, Y. (2006). Learning Absolute Pitch by Children. Music Perception. https://doi.org/10.1525/mp.2006.24.1.63

Mull, H. K. (1925). The acquisition of absolute pitch. Am. J. Psychol, 36, 469-493.

Nosofsky, R. M. (1986). Attention, similarity, and the identificationcategorization relationship. J Exp Psychol Gen, 115(1), 39-61. Retrieved from http://www.ncbi.nlm.nih.gov/htbin-post/Entrez/ query? $\mathrm{db}=\mathrm{m} \&$ form $=6 \&$ dopt $=$ r\&uid $=2937873$

Nosofsky, R. M. (1987). Attention and learning processes in the identification and categorization of integral stimuli. J Exp Psychol Learn Mem Cogn, 13(1), 87-108. Retrieved from http://www.ncbi.nlm.nih. gov/htbin-post/Entrez/query? $\mathrm{db}=\mathrm{m} \&$ form $=6 \& \mathrm{dopt}=\mathrm{r} \&$ uid $=$ 2949055

Oechslin, M. S., Imfeld, A., Loenneker, T., Meyer, M., \& Jäncke, L. (2009). The plasticity of the superior longitudinal fasciculus as a function of musical expertise: a diffusion tensor imaging study. Frontiers in Human Neuroscience, 3(February), 76. https://doi.org/ 10.3389/neuro.09.076.2009

Ohnishi, T., Matsuda, H., Asada, T., Aruga, M., Hirakata, M., Nishikawa, M., ... Imabayashi, E. (2001). Functional anatomy of musical perception in musicians. Cereb Cortex, 11(8), 754-760. Retrieved from http://www.ncbi.nlm.nih.gov/entrez/query.fcgi?db=pubmed\&cmd= Retrieve\&dopt $=$ AbstractPlus\&list_uids $=11459765$

Palmeri, T., \& Gauthier, I. (2004). Visual object understanding. Nat Rev Neurosci, 5(4), 291-303. https://doi.org/10.1038/nrn1364

Pantev, C., Oostenveld, R., Engelien, A., Ross, B., Roberts, L. E., \& Hoke, M. (1998). Increased auditory cortical representation in musicians. Nature, 392(6678), 811-814. https://doi.org/10.1038/33918

Patkowski, M. S. (1990). Age and Accent in a Second Language: A Reply to James Emil Flege. Applied Linguistics, 11(1), 73-89. https://doi.org/10.1093/applin/11.1.73 
Pelli, D. G. (1997). The videotoolbox software for visual psychophysics: transforming numbers into movies. Spatial Vision, 10, 437-442.

Peterson, L. R., \& Peterson, M. J. (1959). Short-term retention of individual items. Journal of Experimental Psychology, 61, 12-21.

Rauscher, F., Shaw, G., Levine, L., Wright, E., Dennis, W., \& Newcomb, R. (1997). Music training causes long-term enhancement of preschool children's spatial-temporal reasoning. Neurological Research, 19(1), 2-8. https://doi.org/10.1080/01616412.1997. 11740765

Rogenmoser, L., Elmer, S., \& Jäncke, L. (2015). Absolute pitch: Evidence for early cognitive facilitation during passive listening as revealed by reduced $\mathrm{P} 3$ a amplitudes. Journal of Cognitive Neuroscience, 27(3), 623-637. https://doi.org/10.1162/jocn a 00708

Ross, D. a., Olson, I. R., Marks, L. E., \& Gore, J. C. (2004). A nonmusical paradigm for identifying absolute pitch possessors. The Journal of the Acoustical Society of America, 116(3), 1793. https://doi.org/10. $1121 / 1.1758973$

Russo, F. A., Windell, D. L., \& Cuddy, L. L. (2003). Learning the "Special Note": Evidence for a Critical Period for Absolute Pitch Acquisition. Music Perception, 21(1), 119-127. https://doi.org/10. 1525/mp.2003.21.1.119

Saffran, J. R., \& Griepentrog, G. J. (2001). Absolute pitch in infant auditory learning: evidence for developmental reorganization. Developmental Psychology, 37(1), 74-85. https://doi.org/10.1037/ 0012-1649.37.1.74

Sakakibara, A. (2014). A longitudinal study of the process of acquiring absolute pitch: A practical report of training with the chord identification method." Psychology of Music, 42(1), 86-111. https://doi. org/10.1177/0305735612463948

Sasaki, Y., Nanez, J. E., \& Watanabe, T. (2010). Advances in visual perceptual learning and plasticity. Nature Reviews. Neuroscience, 11, 53-60. https://doi.org/10.1038/nrn2737

Sathian, K., \& Zangaladze, A. (1997). Tactile learning is task specific but transfers between fingers. Perception \& Psychophysics, 59(1), 119 128. https://doi.org/10.3758/BF03206854

Schellenberg, E. G. (2004). Music lessons enhance IQ. Psychological Science, 15(8), 511-514. https://doi.org/10.1111/j.0956-7976.2004. 00711.x

Schellenberg, E. G., \& Trehub, S. E. (2003). Good pitch memory is widespread. Psychological Science, 14(3), 262-266. Retrieved from http://www.ncbi.nlm.nih.gov/pubmed/12741751

Schlaug, G., Jancke, L., Huang, Y., \& Steinmetz, H. (1995). In vivo evidence of structural brain asymmetry in musicians. Science, 267(5198), 699-701. https://doi.org/10.1126/science.7839149

Schulze, K., Gaab, N., \& Schlaug, G. (2009). Perceiving pitch absolutely: comparing absolute and relative pitch possessors in a pitch memory task. BMC Neuroscience, 10, 106. https://doi.org/10.1186/14712202-10-106

Scovel, T. (1988). A time to speak: A psycholinguistic inquiry into the critical period for human speech. Rowley, MA: Newbury House.

Seitz, A. R., \& Watanabe, T. (2009). The phenomenon of task-irrelevant perceptual learning. Vision Research, 49(21), 2604-2610. https:// doi.org/10.1016/j.visres.2009.08.003

Sengpiel, F. (2007). The critical period. Current Biology, 17(17), R742R743. https://doi.org/10.1016/j.cub.2007.06.017

Simons, D. J., \& Land, P. W. (1987). Early experience of tactile stimulation influences organization of somatic sensory cortex. Nature, 326(6114), 694-697. https://doi.org/10.1038/326694a0

Singleton, D. (2001). Age and second language acquisition. Annual Review of Applied Linguistics, 21, 77-89.

Takeuchi, A H., \& Hulse, S. H. (1993). Absolute pitch. Psychological Bulletin, 113(2), 345-361. Retrieved from http://www.ncbi.nlm.nih. gov/pubmed/8451339

Theusch, E., Basu, A., \& Gitschier, J. (2009). Genome-wide Study of Families with Absolute Pitch Reveals Linkage to 8q24.21 and Locus
Heterogeneity. The American Journal of Human Genetics, 85(1), 112-119. https://doi.org/10.1016/j.ajhg.2009.06.010

Trainor, L. J. (2005). Are there critical periods for musical development? Developmental Psychobiology, 46(3), 262-278. https://doi.org/10. 1002/dev.20059

Tsushima, Y., Sasaki, Y., \& Watanabe, T. (2006). Greater disruption due to failure of inhibitory control on an ambiguous distractor. Science, 314, 1786-1788.

Van Hedger, S. C., Heald, S. L. M., Koch, R., \& Nusbaum, H. C. (2015). Auditory working memory predicts individual differences in absolute pitch learning. Cognition, 140, 95-110. https://doi.org/10.1016/ j.cognition.2015.03.012

Vansteenkiste, M., Simons, J., Lens, W., Sheldon, K. M., \& Deci, E. L. (2004). Motivating learning, performance, and persistence: the synergistic effects of intrinsic goal contents and autonomy-supportive contexts. Journal of Personality and Social Psychology, 87(2), 246260. https://doi.org/10.1037/0022-3514.87.2.246

Vanzella, P., \& Schellenberg, E. G. (2010). Absolute pitch: effects of timbre on note-naming ability. PloS One, 5(11), e15449. https:// doi.org/10.1371/journal.pone.0015449

Ward, J. (2013). Synesthesia. Annual Review of Psychology, 64(1), 4975. https://doi.org/10.1146/annurev-psych-113011-143840

Ward, W. D. (1999). Absolute Pitch. In D. Deutsch (Ed.), The Psychology of Music (pp. 265-298). Academic Press.

Watanabe, T., Nanez, J. E., \& Sasaki, Y. (2001). Perceptual learning without perception. Nature, 413(6858), 844-8. Retrieved from http://www.ncbi.nlm.nih.gov/htbin-post/Entrez/query?db= $\mathrm{m} \&$ form $=6 \&$ dopt $=$ r\&uid $=11677607$

Watanabe, T., \& Sasaki, Y. (2015). Perceptual Learning: Toward a Comprehensive Theory. Annual Review of Psychology, 66(1), 197-221. https://doi.org/10.1146/annurev-psych-010814-015214

Wedell, C. H. (1934). The nature of the absolute judgment of pitch. Journal of Experimental Psychology, 17(4), 485-503. https://doi. org $/ 10.1037 / \mathrm{h} 0070761$

Wengenroth, M., Blatow, M., Heinecke, A., Reinhardt, J., Stippich, C., Hofmann, E., \& Schneider, P. (2013). Increased Volume and Function of Right Auditory Cortex as a Marker for Absolute Pitch. Cerebral Cortex, 24(May), 1127-1137. https://doi.org/10.1093/ cercor/bhs391

West Marvin, E., VanderStel, J., \& Siu, J. C.-S. (2019). In their own words: Analyzing the extents and origins of absolute pitch. Psychology of Music, 030573561983295. https://doi.org/10.1177/ 0305735619832959

Wiesel, T. N., \& Hubel, D. H. (1963). Effects of visual deprivation on morphology and physiology of cells in the cat's lateral geniculate body. Journal of Neurophysiology, 26(6), 978-993. https://doi.org/ 10.1152/jn.1963.26.6.978

Wilson, D. A., \& Stevenson, R. J. (2003). Olfactory perceptual learning: the critical role of memory in odor discrimination. Neuroscience \& Biobehavioral Reviews, 27(4), 307-328. https://doi.org/10.1016/ S0149-7634(03)00050-2

Wilson, S. J., Lusher, D., Wan, C. Y., Dudgeon, P., \& Reutens, D. C. (2009). The neurocognitive components of pitch processing: insights from absolute pitch. Cerebral Cortex (New York, N.Y. : 1991), 19(3), 724-732. https://doi.org/10.1093/cercor/bhn121

Wong, A. C.-N., Palmeri, T., \& Gauthier, I. (2009). Conditions for facelike expertise with objects: Becoming a Ziggerin expert - but which type? Psychological Science, 20(9), 1108-1117.

Wong, Y. K., Folstein, J. R., \& Gauthier, I. (2011). Task-irrelevant perceptual expertise. Journal of Vision. https://doi.org/10.1167/11.14.3

Wong, Y. K., \& Wong, A. C.-N. (2014). Absolute pitch memory: Its prevalence among musicians and dependence on the testing context. Psychonomic Bulletin \& Review, 21, 534-542. https://doi.org/10. 3758/s13423-013-0487-z

Wright, B. A., Buonomano, D. V, Mahncke, H. W., \& Merzenich, M. M. (1997). Learning and generalization of auditory temporal-interval 
discrimination in humans. Journal of Neuroscience, 17(10), 39563963.

Wu, C., Kirk, I. J., Hamm, J. P., \& Lim, V. K. (2008). The neural networks involved in pitch labeling of absolute pitch musicians. Neuroreport, $19(8), 851-854$.

Zatorre, R. J. (2003). Absolute pitch: a model for understanding the influence of genes and development on neural and cognitive function. Nature Neuroscience, 6(7), 692-695. https://doi.org/10.1038/ nn1085

Zatorre, R. J., Perry, D. W., Beckett, C. A., Westbury, C. F., \& Evans, A. C. (1998). Functional anatomy of musical processing in listeners with absolute pitch and relative pitch. Proceedings of the National Academy of Sciences of the United States of America, 95(6), 31723177.
Zeanah, C. H., Gunnar, M. R., McCall, R. B., Kreppner, J. M., \& Fox, N. A. (2011). VI. SENSITIVE PERIODS. Monographs of the Society for Research in Child Development, 76(4), 147-162. https://doi.org/ 10.1111/j.1540-5834.2011.00631.x

Zhang, L. I., Bao, S., \& Merzenich, M. M. (2002). Disruption of primary auditory cortex by synchronous auditory inputs during a critical period. Proceedings of the National Academy of Sciences, 99(4), 2309-2314. https://doi.org/10.1073/pnas.261707398

Publisher's note Springer Nature remains neutral with regard to jurisdictional claims in published maps and institutional affiliations. 